\title{
Infall and Outflow of Molecular Gas in Sgr B2
}

\section{Citation}

Qin, Sheng\#Li, Jun\#Hui Zhao, James M. Moran, Daniel P. Marrone, Nimesh A. Patel, Jun\# Jie Wang, Sheng\#Yuan Liu, and Yi\#Jehng Kuan. 2008. "Infall and Outflow of Molecular Gas in Sgr B2." Astrophysical Journal 677 (1) (April 10): 353-372. doi:10.1086/529067.

\section{Published Version}

doi:10.1086/529067

\section{Permanent link}

http://nrs.harvard.edu/urn-3:HUL.InstRepos:11596972

\section{Terms of Use}

This article was downloaded from Harvard University's DASH repository, and is made available under the terms and conditions applicable to Other Posted Material, as set forth at http:// nrs.harvard.edu/urn-3:HUL.InstRepos:dash.current.terms-of-use\#LAA

\section{Share Your Story}

The Harvard community has made this article openly available.

Please share how this access benefits you. Submit a story.

Accessibility 


\title{
INFALL AND OUTFLOW OF MOLECULAR GAS IN SGR B2
}

\author{
Sheng-Li Qin, ${ }^{1,2}$ Jun-Hui Zhao, ${ }^{1}$ James M. Moran, ${ }^{1}$ Daniel P. Marrone, ${ }^{1}$ Nimesh A. Patel, ${ }^{1}$ \\ Jun-JiE Wang, ${ }^{2}$ Sheng-YuAn LiU, ${ }^{3}$ and Yi-Jehng KuAN ${ }^{3,4}$ \\ Received 2007 September 20; accepted 2008 January 1
}

\begin{abstract}
Observations of two $\mathrm{H}_{2} \mathrm{CO}\left(3_{03}-2_{02}\right.$ and $\left.3_{21}-2_{20}\right)$ lines and continuum emission at $1.3 \mathrm{~mm}$ toward $\mathrm{Sgr} \mathrm{B} 2(\mathrm{~N})$ and Sgr B2(M) have been carried out with the SMA. The mosaic maps of Sgr B2(N) and Sgr B2(M) in both continuum and lines show a complex distribution of dust and molecular gas in both clumps and filaments surrounding the compact star formation cores. We have observed a decelerating outflow originated from the Sgr B2(M) core, showing that both the redshifted and blueshifted outflow components have a common terminal velocity. This terminal velocity is $58 \pm 2 \mathrm{~km} \mathrm{~s}^{-1}$. It provides an excellent method for determination of the systematic velocity of the molecular cloud. The SMA observations have also shown that a large fraction of absorption against the two continuum cores is redshifted with respect to the systematic velocities of Sgr B2(N) and Sgr B2(M), respectively, suggesting that the majority of the dense molecular gas is flowing into the two major cores where massive stars have been formed. We have solved the radiative transfer in a multilevel system with LVG approximation. The observed $\mathrm{H}_{2} \mathrm{CO}$ line intensities and their ratios can be adequately fitted with this model for the most of the gas components. However, the line intensities between the higher energy level transition $\mathrm{H}_{2} \mathrm{CO}\left(3_{21}-2_{20}\right)$ and the lower energy level transition $\mathrm{H}_{2} \mathrm{CO}\left(3_{03}-2_{02}\right)$ is reversed in the redshifted outflow region of Sgr B2(M), suggesting the presence of inversion in population between the ground levels in the two $K$ ladders $\left(K_{-1}=0\right.$ and 2$)$. The possibility of weak maser processes for the $\mathrm{H}_{2} \mathrm{CO}$ emission in Sgr B2(M) is discussed.
\end{abstract}

Subject headings: Galaxy: center — ISM: individual (Sgr B2) — ISM: kinematics and dynamics — ISM: molecules — radio lines: ISM — stars: formation

Online material: color figure

\section{INTRODUCTION}

The giant molecular cloud Sgr B2, located close to the Galactic center $\left(\sim 44^{\prime}\right.$ from Sgr $\left.A^{*}\right)$, is a well-known massive starforming region in our Galaxy. Sgr B2 consists of an extended envelope, a hot ring, and a few compact cores (e.g., Goicoechea et al. 2004). The radio continuum and recombination line observations of the compact $\mathrm{H}$ II regions suggest that $\mathrm{Sgr} B 2(\mathrm{~N})$ and Sgr B2 (M) are the two most active star-forming cores in this region (Gaume \& Claussen 1990; Gaume et al. 1995; Mehringer et al. 1993; de Pree et al. 1995, 1996, 1998). Masers, outflows, and possible rotation of the two dense cores have been revealed from observations of various molecular lines at centimeter and millimeter wavelengths (Reid et al. 1988; Gaume \& Claussen 1990; Martin-Pintado et al. 1990; Mehringer et al. 1994; Lis et al. 1993; Kuan \& Snyder 1996; Liu \& Snyder 1999). In addition, previous observations have shown evidence for the two hot cores to be at different evolutionary stages and to have different molecular abundances (e.g., Vogel et al. 1987; Lis et al. 1993; Miao et al. 1995; Kuan et al. 1996; Liu \& Snyder 1999).

$\mathrm{H}_{2} \mathrm{CO}$ pervades the interstellar medium and it has a simple chemical reaction path which has been proven to be a useful probe of physical conditions (e.g., Mangum \& Wootten 1993). The $\mathrm{H}_{2} \mathrm{CO}\left(1_{10}-1_{11}\right)$ transition at $6 \mathrm{~cm}$ was observed in absorption against discrete continuum sources toward Sgr B2 complex

\footnotetext{
1 Harvard-Smithsonian Center for Astrophysics, 60 Garden Street, MS 42, Cambridge, MA 02138; sqin@cfa.harvard.edu.

2 National Astronomical Observatories, Chinese Academy of Sciences, Beijing, 100012, China.

3 Academia Sinica Institute of Astronomy and Astrophysics, P.O. Box 23-141, Taipei 106, Taiwan.

4 Department of Earth Sciences, National Taiwan Normal University, 88 Section 4, Ting-Chou Road, Taipei 116, Taiwan.
}

with an angular resolution of $\sim 10^{\prime \prime} \times 20^{\prime \prime}$, showing nearly the same radial velocity pattern as that of the radio recombination lines (Martin-Pintado et al. 1990; Mehringer et al. 1995). These authors suggested that the $\mathrm{H}_{2} \mathrm{CO}\left(1_{10}-1_{11}\right)$ transition probably arises from the surrounding gas with a relatively low mean $\mathrm{H}_{2}$ density of $\sim 10^{4} \mathrm{~cm}^{-3}$ (Martin-Pintado et al. 1990; Mehringer et al. 1995).

The millimeter $\mathrm{H}_{2} \mathrm{CO}$ lines are an excellent tracer of $\mathrm{H}_{2}$ density $>10^{5} \mathrm{~cm}^{-3}$ (e.g., Mangum \& Wootten 1993). In addition, $\mathrm{H}_{2} \mathrm{CO}$ is a planar asymmetric top molecule with very little asymmetry. The symmetry of the spin function of the molecule leads to two transition classes: ortho- $\mathrm{H}_{2} \mathrm{CO}$ levels if the spin wave function is symmetric and para- $\mathrm{H}_{2} \mathrm{CO}$ levels if antisymmetric. Since para$\mathrm{H}_{2} \mathrm{CO}$ is $1-3$ times less abundant than ortho- $\mathrm{H}_{2} \mathrm{CO}$, observations of para- $\mathrm{H}_{2} \mathrm{CO}$ have less opacity effect (Kahane et al. 1984; Mangum \& Wootten 1993). Hence, para- $\mathrm{H}_{2} \mathrm{CO}$ appears to be a better probe to determine the physical conditions of the massive star formation regions.

The millimeter/submillimeter transitions of $\mathrm{H}_{2} \mathrm{CO}$ gas require relatively high excitation temperature and high $\mathrm{H}_{2}$ density compared to those in the centimeter wave bands. If the brightness temperature of the continuum emission is higher than the excitation temperature, the absorption against the continuum cores can be observed in millimeter and submillimeter wave bands with the high angular resolution of an interferometric array (such as the Submillimeter Array, ${ }^{5}$ hereafter SMA). Taking advantage of the large bandwidth coverage of the SMA, we have observed multiple $\mathrm{H}_{2} \mathrm{CO}$ lines toward Sgr B2 at $1.3 \mathrm{~mm}$ within a bandwidth of

\footnotetext{
5 The Submillimeter Array is a joint project between the Smithsonian Astrophysical Observatory and the Academia Sinica Institute of Astronomy and Astrophysics and is funded by the Smithsonian Institution and the Academia Sinica.
} 
$2 \mathrm{GHz}$. Thus, with the same telescope system and calibration procedure, the uncertainties due to absolute flux density calibration among the different line transitions can be mitigated by measuring the line-intensity ratios, which are needed to determine physical conditions, such as kinetic temperature and $\mathrm{H}_{2}$ number density, of the gas. In addition, the SMA is not sensitive to extended larger scale emission $\left(\sim 50^{\prime \prime}\right)$. Thus, the SMA observations are sensitive to the clumps of high-density gas rather than the extended diffuse components.

In this paper, we present the results from the SMA observations of $\mathrm{Sgr} \mathrm{B} 2$ at the $\mathrm{H}_{2} \mathrm{CO}$ lines and continuum at $1.3 \mathrm{~mm}$. The paper is organized as follows: $\S 2$ discusses the observations and data reduction. In $\S 3$ we present the data analysis and results. In $\S 4$, we present the kinematics in Sgr B2(M) by a model incorporating a spherically symmetric inflow along with a decelerating outflow. In $\S 5$ we model the physical properties of the $\mathrm{H}_{2} \mathrm{CO}$ gas in $\mathrm{Sgr} \mathrm{B} 2$ using the large velocity gradient (LVG) approach. In $\S 6$, we discuss the important results derived from our observations and analysis. We summarize the results in $\S 7$. We adopt a distance of $8 \mathrm{kpc}$ to Sgr B2.

\section{OBSERVATIONS AND DATA REDUCTION}

Observations toward Sgr B2(N) and Sgr B2(M) were carried out at 218 (lower sideband) and $228 \mathrm{GHz}$ (upper sideband) with seven antennas of the SMA in the compact north array on 2005 August 1 for $8 \mathrm{hr}$. The projected baselines ranged from 5 to $50 \mathrm{k} \lambda$. The weather was good during the observations with $\tau \sim$ 0.09 at $225 \mathrm{GHz}$. The typical system temperature was $130 \mathrm{~K}$. Sgr B2(N) and Sgr B2(M) were observed in separate fields with 15 minute observing times on each source interleaving 5 minutes on the phase reference source $\operatorname{Sgr~A}^{*}(<0.1$ mas, $\sim 3$ Jy at $230 \mathrm{GHz}, \sim 45^{\prime}$ from Sgr B2). Our two target fields were centered at R.A.(J2000.0) $=17 \mathrm{~h}^{\mathrm{h}} 47^{\mathrm{m}} 19.882^{\mathrm{s}}$, decl.(J2000.0) $=$ $-28^{\circ} 22^{\prime} 18.37^{\prime \prime} \quad$ and R.A.(J2000.0) $=17^{\mathrm{h}} 47^{\mathrm{m}} 20.156^{\mathrm{s}}$, decl.(J2000.0) $=-28^{\circ} 23^{\prime} 03.56^{\prime \prime}$, for Sgr B2(N) and Sgr B2(M), respectively. In addition, Callisto (4.1 Jy) and the QSO 3C454.3 (32 Jy) were also observed for the flux-density and bandpass calibrations. The flux density was estimated from Callisto with the assumption that its brightness temperature was $120 \mathrm{~K}$ and its angular size was $1.14^{\prime \prime}$. The three transitions $\mathrm{H}_{2} \mathrm{CO}\left(3_{03}-2_{02}\right),\left(3_{22}-2_{21}\right)$, and $\left(3_{21}-2_{20}\right)$ were positioned in the lower $2 \mathrm{GHz}$ sideband (LSB). $\mathrm{H}_{2} \mathrm{CO}\left(3_{22}-2_{21}\right)$ emission appears to be blended with a transition of $\mathrm{CH}_{3} \mathrm{OH}$ and will be not used in the analysis in this paper. The two unblended transitions, $\mathrm{H}_{2} \mathrm{CO}\left(3_{03}-2_{02}\right)$ and $\left(3_{21}-2_{20}\right)$, have upper level energies of 21.0 and $67.8 \mathrm{~K}$ and rest frequencies of 218.2222 and $218.7601 \mathrm{GHz}$, respectively. The spectral resolution of $0.8125 \mathrm{MHz}$ corresponds to a velocity resolution of $1.1 \mathrm{~km} \mathrm{~s}^{-1}$.

The data reduction was carried out in Miriad. ${ }^{6}$ We recomputed the Doppler velocity for each of the target sources since the online Doppler tracking was only made on Sgr B2(N). System temperature corrections were applied. Antenna-based bandpass ripples were corrected by applying the linear interpolation of the bandpass solutions determined from Callisto and 3C454.3. There are spectral-window-based offsets in amplitude and phase on some baselines due to the correlator errors, which were also corrected. The residual errors due to the bandpass shape of the individual spectral windows were reduced to a level below $1 \%$ of the continuum level. In the antenna-based gain corrections, we chose the visibilities of $\mathrm{Sgr} \mathrm{A}^{*}$ in the UV range between 20 kilowavelengths and longer in order to eliminate the contamination

\footnotetext{
${ }^{6}$ The data reduction procedures for SMA data are outlined in the Web site http://sma-www.cfa.harvard.edu/miriadWWW.
}

from the extended dust and $\mathrm{H}$ II emission. The gains determined from the point source (emission from $\mathrm{Sgr} \mathrm{A}^{*}$ ) were applied to the Sgr B2 data.

The molecular lines were identified in the rest frame by use of the JPL catalog, as done by others (Sutton et al. 1985; Nummelin et al. 1998). The $\mathrm{H}_{2} \mathrm{CO}$ transitions and a few other molecular lines have been unambiguously identified (see spectrum in Fig. 1).

The continuum was subtracted with a linear fitting to the spectrum of line-free channels in each baseline. For Sgr B2(M), the line-free channels can be easily selected from the spectrum in the $(u, v)$ domain (Fig. 1). However, for Sgr B2(N), the molecular spectral lines are crowded over each $2 \mathrm{GHz}$ band and it is difficult to choose the line-free channels. We developed a procedure to select the line-free channels for the continuum subtraction. First, after reducing the spectral resolution to $1 \mathrm{~km} \mathrm{~s}^{-1}$, we made each of the channel maps (2600 channels over the $2 \mathrm{GHz}$ band) including both the line and continuum. From the channel maps, we selected the channels without extended emission $(>4 \sigma)$. The continuum level was determined from these apparently line-free channels. We selected 16 and 37 line-free spectral windows for the fields of Sgr B2(M) and Sgr B2(N), respectively. Then, using the task UVLIN in Miriad, we determined the continuum level by fitting to the line-free channels. The UVLIN gives two output $(u, v)$ data sets, one for the continuum and the other for continuumfree spectral line.

Self-calibration was performed to the continuum data using the compact sources of the continuum cores for a few iterations in order to eliminate residual errors. The gain solutions determined from the continuum data were applied to the line data, from which we constructed the spectral data cube. The preliminary images of the continuum and lines were made using natural weighting. The clean algorithm was applied to remove the effects of the side lobes. The synthesized beam sizes of the continuum and line images were approximately $5.4^{\prime \prime} \times 3.2^{\prime \prime}\left(\right.$ P.A. $\left.=12.5^{\circ}\right)$. The mosaic maps of the two fields, Sgr B2(N) and Sgr B2(M), were made using a simple linear mosaic algorithm. The primary beam attenuation was also corrected in the final line cubes and continuum image. The statistical $1 \sigma \mathrm{rms}$ noise of the continuum was $0.1 \mathrm{Jy}$ beam $^{-1}$. The $1 \sigma \mathrm{rms}$ noise levels of the line images were 0.18 and $0.16 \mathrm{Jy}$ beam $^{-1}$ per channel for the $\mathrm{H}_{2} \mathrm{CO}\left(3_{03}-2_{02}\right)$ and $\left(3_{21}-2_{20}\right)$ lines, respectively. The higher noise in the $\mathrm{H}_{2} \mathrm{CO}\left(3_{03}-2_{02}\right)$ line images was due to its strong line intensity and the limit of the dynamic range in the clean process.

\section{DATA ANALYSIS AND RESULTS}

\subsection{The Continuum at $1.3 \mathrm{~mm}$}

Figure 2 shows the mosaic continuum map of Sgr B2(N) and Sgr B2(M) at $1.3 \mathrm{~mm}$. The brightest components of Sgr B2(N) $\left(I_{p}=29.2 \pm 2.1 \mathrm{Jy}\right.$ beam $\left.^{-1}\right)$ and Sgr B2(M) $\left(I_{p}=20.2 \pm\right.$ $\left.1.3 \mathrm{Jy} \mathrm{beam}^{-1}\right)$, are associated with the massive star-forming cores K1-3 and F1-4 (Gaume \& Claussen 1990), respectively. In addition to the emission from these cores, a few nearby continuum clumps were detected, including the components K4 $\left(I_{p}=\right.$ $\left.1.40 \pm 0.12 \mathrm{Jy} \mathrm{beam}^{-1}\right), \mathrm{NE}\left(I_{p}=1.20 \pm 0.14 \mathrm{Jy} \mathrm{beam}^{-1}\right)$, NW $\left(I_{p}=1.00 \pm 0.11 \mathrm{Jy} \mathrm{beam}^{-1}\right), \mathrm{ME}\left(I_{p}=0.58 \pm 0.15 \mathrm{Jy} \mathrm{beam}^{-1}\right)$, and MW $\left(I_{p}=1.70 \pm 0.18 \mathrm{Jy}\right.$ beam $\left.^{-1}\right)$.

Gaussian fitting to the individual continuum components was carried out. The individual emission clumps near the compact cores or the K1-3 and F1-4 clusters were modeled as simple Gaussian components. The two compact cores, K1-3 and F1-4, appeared to be too complicated to be fitted with single Gaussian components. A model consisting of a Gaussian and two unresolved compact components was used to fit to the data of the core (K1-3). The 


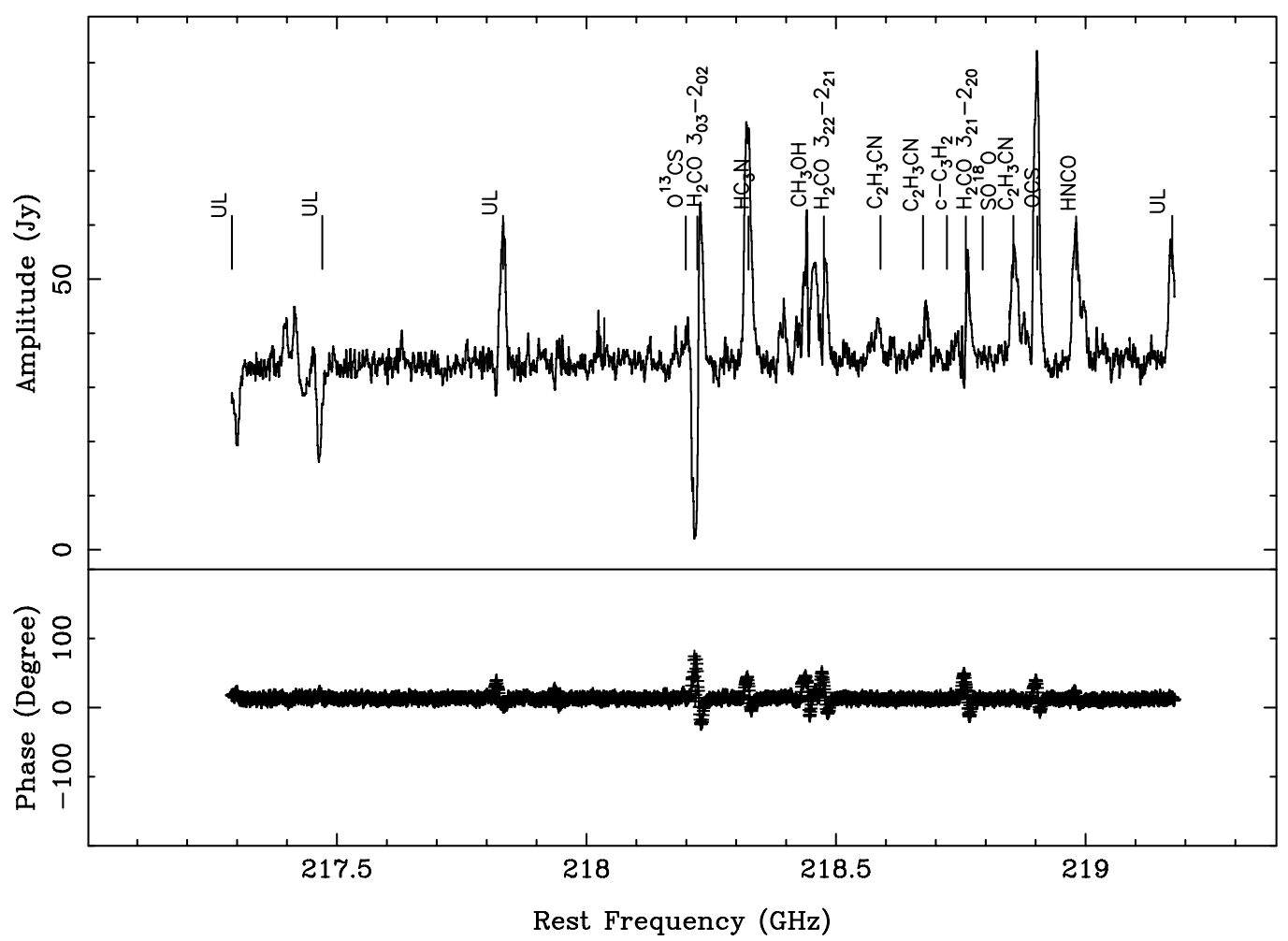

Fig. 1.-Spectrum from $(u, v)$ domain on the baseline 3-4 for $\mathrm{Sgr} \mathrm{B} 2(\mathrm{M})$. The horizontal axis is the frequency in the rest frame and the vertical is the amplitude and phase. UL indicates the unidentified lines. We note that the features at 218.222 and $218.760 \mathrm{GHz}$ are apparently dominated by $\mathrm{H}_{2} \mathrm{CO}\left(3_{03}-2_{02}\right.$ and $\left.3_{21}-2_{20}\right)$ and the possible contaminations from $\mathrm{O}^{13} \mathrm{CS}, \mathrm{c}_{-} \mathrm{C}_{3} \mathrm{H}_{2}$, and $\mathrm{SO}^{18} \mathrm{O}$ at the transitions near the $\mathrm{H}_{2} \mathrm{CO}$ frequencies 218.222 and $218.760 \mathrm{GHz}$ appear to be insignificant in Sgr B2(M). These molecular lines also appear to be insignificant in $\mathrm{Sgr} \mathrm{B} 2(\mathrm{~N})$. [See the electronic edition of the Journal for a color version of this figure.]

peaks of the Gaussian component and one of the point components are consistent with K3 and K2 positions, respectively. Another point component agrees (within $\sim 1^{\prime \prime}$ ) with the $3.5 \mathrm{~mm}$ continuum source that is located south of K3 (see Fig. $1 b$ of Liu \& Snyder 1999). The Sgr B2(M) core (F1-4) is fitted well with a Gaussian component and a point component (close to F3 within $\sim 0.5^{\prime \prime}$ ). The peak positions, deconvolved angular sizes, peak intensities, and total flux densities of the continuum components are summarized in Table 1.

The components K4, MW, and Z10.24 have been detected at radio and millimeter wavelengths (Lis et al. 1993; Kuan \& Snyder 1994; Gaume et al. 1995; Liu \& Snyder 1999). Z10.24, located in the middle between Sgr B2(N) and Sgr B2(M) (see Fig. 2), shows a unique filamentary structure at $1.3 \mathrm{~mm}$ continuum. The designation of Z10.24 follows that used by Gaume et al. (1995) and de Pree et al. (1996), who detected the H66 $\alpha$ line toward it. Z10.24 was marginally detected at $1.3 \mathrm{~mm}$ by Lis et al. (1993) with higher angular resolution $\left(4.5^{\prime \prime} \times 3.7^{\prime \prime}\right)$ and poorer sensitivity. The SMA observations show an elongated structure in $\mathrm{Z} 10.24$ with a peak intensity of $2.02 \pm 0.16 \mathrm{Jy} \mathrm{beam}^{-1}(12 \sigma)$. The core of Z10.24 is unresolved in the subarcsecond resolution images at both $1.3 \mathrm{~cm}$ and $3.5 \mathrm{~mm}$ (Gaume et al. 1995; Liu \& Snyder 1999). The $1.3 \mathrm{~mm}$ continuum image and the detected vibrational $\mathrm{HC}_{3} \mathrm{~N}$ emission (de Vicente et al. 2000) suggest that the $1.3 \mathrm{~mm}$ continuum of $\mathrm{Z} 10.24$ is dominated by the dust emission and Z10.24 is likely to be a younger massive star formation region.

There have been no detections of the components NW, NE, and $\mathrm{ME}$ in the previous observations at longer wavelengths. These three components are possibly the dust emission from subcores at a relatively early stage of star formation. The detections need to be verified with further observations at shorter wavelengths and at higher angular resolutions.

\section{2. $\mathrm{H}_{2} \mathrm{CO}$ Lines}

The continuum-free channel maps in both $\mathrm{H}_{2} \mathrm{CO}\left(3_{03}-2_{02}\right)$ and $\mathrm{H}_{2} \mathrm{CO}\left(3_{21}-2_{20}\right)$ lines were constructed in the velocity range from 8 to $151 \mathrm{~km} \mathrm{~s}^{-1}$ at intervals of $1 \mathrm{~km} \mathrm{~s}^{-1}$. The channel maps of the $\mathrm{H}_{2} \mathrm{CO}$ transitions are complicated, containing several kinematical features in either emission or absorption. Those emission and absorption components are separated well in our higher spectral resolution maps but some of them (in the continuum core regions) are overlapped. In the moment analysis, the negative intensity value from the absorption and the positive value from the emission may cancel each other in the overlapping regions and thus the resultant moment maps might not reflect the true gas distribution. Hence, the emission and absorption need to be handled separately.

\subsubsection{Line Emission}

Figure 3 is the integrated line emission images constructed from the channel maps for the two transitions, $\mathrm{H}_{2} \mathrm{CO}\left(3_{21}-2_{20}\right)$ and $\left(3_{03}-2_{02}\right)$, respectively. The moment 0 images were made with a $4 \sigma$ cutoff in each channel map. The less significant emission and the absorption are excluded in the moment analysis. Most of emission is distributed around the two cores of Sgr B2(N) and Sgr B2(M). Clearly, the distribution of the $\mathrm{H}_{2} \mathrm{CO}$ emission is not spherically symmetric with respect to each of these cores. In the Sgr B2(N) region, in addition to the gas concentration at the core, gas clumps located northeast and southwest of the core are observed in both 


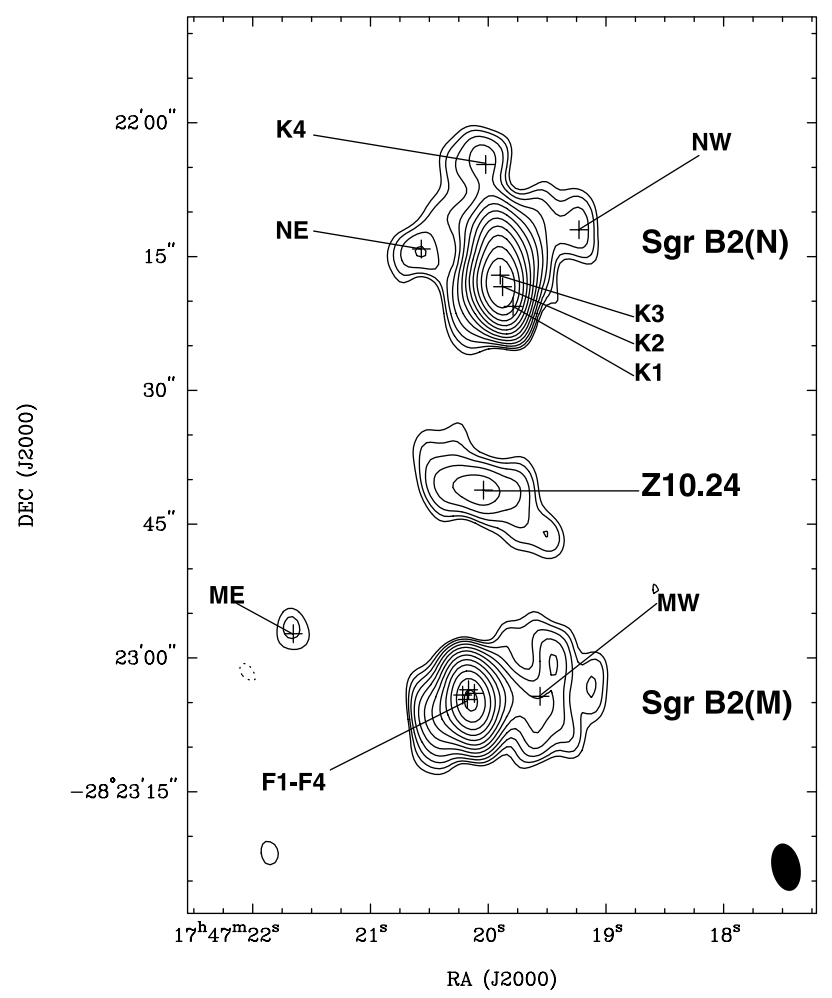

FIG. 2.-Mosaic image of the continuum emission toward Sgr B2 at $1.3 \mathrm{~mm}$ obtained by combining the data from both sidebands $(218$ and $228 \mathrm{GHz})$. The synthesized beam is $5.4^{\prime \prime} \times 3.2^{\prime \prime}$, P.A. $=12.5^{\circ}$ (lower right corner). The contours are $-4,4,5.6,8,11,16,23,32,45,64,91,128$, and $181 \sigma$. The rms $(1 \sigma)$ noise level is $0.1 \mathrm{Jy}$ beam $^{-1}$. The plus symbols indicate the positions of the continuum sources at $1.3 \mathrm{~mm}$. The $\mathrm{UCH}$ in region $\mathrm{K} 1-3$ and $\mathrm{F} 1-4$ positions were taken from Gaume et al. (1995).

$\mathrm{H}_{2} \mathrm{CO}$ transitions. We note that in Sgr B2(N), the morphology of $\mathrm{H}_{2} \mathrm{CO}\left(3_{21}-2_{20}\right)$ emission, the higher transition gas, is similar to that of the emission from the lower transition gas $\mathrm{H}_{2} \mathrm{CO}\left(3_{03}-2_{02}\right)$. Hereafter we refer to $\mathrm{H}_{2} \mathrm{CO}\left(3_{21}-2_{20}\right)$ as the higher transition and $\mathrm{H}_{2} \mathrm{CO}\left(3_{03}-2_{02}\right)$ as the lower transition. In the Sgr B2(M) region, a strong emission component elongated in the northwestsoutheast direction is observed in both $\mathrm{H}_{2} \mathrm{CO}$ transitions. An arch structure (M1 30" long and 10" wide), $8^{\prime \prime}$ northwest of the Sgr B2(M) core appears in both $\mathrm{H}_{2} \mathrm{CO}$ transitions. The major difference in the emission distribution from the two transitions occurs in the outflow (Lis et al. 1993) region located southeast of the Sgr B2(M) core. A significant emission "tongue" (M5) $\left(15^{\prime \prime} \times 7^{\prime \prime}\right)$ was detected from the higher $\mathrm{H}_{2} \mathrm{CO}$ transition gas, while no significant detection was made of the lower $\mathrm{H}_{2} \mathrm{CO}$ transition gas.

\subsubsection{Systematic Velocities}

Based on a line survey at $340 \mathrm{GHz}$ from single-dish observations, Sutton et al. (1991) obtained mean systematic velocities 65 and $61 \mathrm{~km} \mathrm{~s}^{-1}$ of Sgr B2(N) and Sgr B2(M). Their observations showed significant velocity variations among the different species. The differences are mostly caused by the chemical differences of the molecules. The different species sample different physical environments. The high angular resolution observations of H66 $\alpha$ (de Pree et al. 1995, 1996) showed that the mean systematic velocities are 69.8 and $65.3 \mathrm{~km} \mathrm{~s}^{-1}$ for the Sgr B2(M)-F and Sgr B2(N)-K clusters, respectively. The relatively higher mean velocity of $69.8 \mathrm{~km} \mathrm{~s}^{-1}$ in Sgr B2(M) is likely caused by the highvelocity motion of the ionized gas of the $\mathrm{UCH}$ II regions with respect to the centroid of the system. In $\S 4$, we will show that the systematic velocity determined from the terminal velocity of the outflow in Sgr B2(M) is $58 \mathrm{~km} \mathrm{~s}^{-1}$ (see also Appendix A). In the rest of the paper, we adopt systematic velocities of 58 and $65 \mathrm{~km} \mathrm{~s}^{-1}$ for Sgr B2(M) and Sgr B2(N), respectively.

\subsubsection{Absorption toward the Cores}

The strong continuum cores are excellent probes of absorption by the cold gas that resides in front of them. Absorption is observed toward both the Sgr B2(N) and Sgr B2(M) continuum cores (see Fig. 3, left). Multiple Gaussian line components were fitted to the spectra for each of the two transitions in both cores. The parameters of these fits are listed in Table 2. The systematic velocities are marked with vertical lines in Figure 3. The majority of the absorbing gas in Sgr B2(N) and Sgr B2(M) is redshifted with respect to the systematic velocities 58 and $65 \mathrm{~km} \mathrm{~s}^{-1}$, respectively. The redshifted absorption gas provides evidence for the existence of gas accreting onto the two cores.

If the absorbing gas covers the continuum source completely and the emission from the gas is insignificant, the line intensity is

TABLE 1

The SMa Measurements of Continuum Emission

\begin{tabular}{|c|c|c|c|c|c|c|c|}
\hline Source & R.A. (J2000.0) & $\begin{array}{c}\Delta \text { R.A. } \\
(\operatorname{arcsec})\end{array}$ & Decl. (J2000.0) & $\begin{array}{l}\Delta \text { Decl. } \\
(\operatorname{arcsec})\end{array}$ & Angular Size and (PA) & $\begin{array}{l}\text { Peak Intensity } \\
\left(\text { Jy beam }^{-1}\right)\end{array}$ & $\begin{array}{c}\text { Flux Density } \\
(\mathrm{Jy})\end{array}$ \\
\hline $\mathrm{K} 1-3^{\mathrm{a}} \ldots \ldots$ & 174719.89 & \pm 0.1 & -282217.6 & \pm 0.3 & $6.8^{\prime \prime} \times 3.5^{\prime \prime}\left(-25^{\circ}\right)$ & $29.2 \pm 2$ & $52.1 \pm 4$ \\
\hline K4 & 174720.02 & \pm 0.1 & $\begin{array}{lll}-28 & 22 & 04.7\end{array}$ & \pm 0.1 & $5.2^{\prime \prime} \times 4.8^{\prime \prime}\left(-49^{\circ}\right)$ & $1.4 \pm 0.1$ & $3.5 \pm 0.3$ \\
\hline NE & 174720.56 & \pm 0.5 & -282214.4 & \pm 0.3 & $5.9^{\prime \prime} \times 1.4^{\prime \prime}\left(-71^{\circ}\right)$ & $1.2 \pm 0.1$ & $2.5 \pm 0.3$ \\
\hline NW & 174719.27 & \pm 0.3 & -282211.8 & \pm 0.3 & $5.1^{\prime \prime} \times 4.1^{\prime \prime}\left(0^{\circ}\right)$ & $1.0 \pm 0.1$ & $2.3 \pm 0.2$ \\
\hline 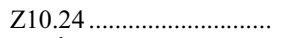 & 174720.04 & \pm 0.3 & -282241.2 & \pm 0.4 & $13.3^{\prime \prime} \times 4.4^{\prime \prime}\left(66^{\circ}\right)$ & $2.0 \pm 0.2$ & $9.1 \pm 0.8$ \\
\hline 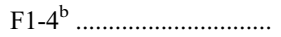 & 174720.17 & \pm 0.2 & -282304.9 & \pm 0.3 & $7.8^{\prime \prime} \times 4.4^{\prime \prime}\left(-58^{\circ}\right)$ & $20.2 \pm 1.3$ & $35.6 \pm 3$ \\
\hline 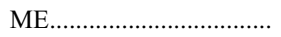 & 174721.65 & \pm 0.6 & -282257.0 & \pm 0.7 & $4.1^{\prime \prime} \times 2.6^{\prime \prime}\left(73^{\circ}\right)$ & $0.58 \pm 0.15$ & $1.0 \pm 0.3$ \\
\hline 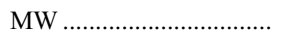 & 174719.56 & \pm 0.8 & -282304.3 & \pm 0.6 & $12.8^{\prime \prime} \times 8.3^{\prime \prime}\left(-13^{\circ}\right)$ & $1.7 \pm 0.2$ & $12.3 \pm 1.3$ \\
\hline
\end{tabular}

NoтE.- Units of right ascension are hours, minutes, and seconds, and units of declination are degrees, arcminutes, and arcseconds.

${ }^{\text {a }}$ Sgr B2(N) compact core (K1-3) was fitted with a Gaussian source $\left(6.8^{\prime \prime} \times 3.5^{\prime \prime}\right.$, P.A. $\left.=-24.8^{\circ}\right)$ centered on K3 [R.A. $(\mathrm{J} 2000.0)=17^{\mathrm{h}} 47^{\mathrm{m}} 19.90^{\mathrm{s}}$, decl. $(\mathrm{J} 2000.0)=$ $-28^{\circ} 22^{\prime} 16.9^{\prime \prime}$ ] and two point sources at K2 [R.A.(J2000.0) $=17^{\mathrm{h}} 47^{\mathrm{m}} 19.88^{\mathrm{s}}$, decl.(J2000.0) $\left.=-28^{\circ} 22^{\prime} 18.6^{\prime \prime}\right]$ and at $\left[\right.$ R.A.(J2000.0) $=17^{\mathrm{h}} 47^{\mathrm{m}} 19.95^{\mathrm{s}}$, decl.(J2000.0) $=$ $\left.-28^{\circ} 22^{\prime} 12.3^{\prime \prime}\right]$. The flux densities of the Gaussian source and the two point sources are $29.4 \pm 3.7,19.1 \pm 1.5$, and $3.6 \pm 1.0 \mathrm{Jy}$, respectively. The total flux density of the compact core (K1-3) is $52.1 \pm 4 \mathrm{Jy}$.

${ }^{b}$ Sgr B2(M) compact core (F1-4) was fitted with a Gaussian source $\left(7.8^{\prime \prime} \times 4.4^{\prime \prime}\right.$, P.A. $\left.=-58.1^{\circ}\right)$ located within F cluster at $\left[\right.$ R.A. $(\mathrm{J} 2000.0)=17^{\mathrm{h}} 47^{\mathrm{m}} 20.17^{\mathrm{s}}$, decl.(J2000.0) $=-28^{\circ} 23^{\prime} 05.1^{\prime \prime}$ ] and a point source centered on F3 [R.A.(J2000.0) $=17^{\mathrm{h}} 47^{\mathrm{m}} 20.14^{\mathrm{s}}$, decl.(J2000.0) $=-28^{\circ} 23^{\prime} 04.6^{\prime \prime}$ ]. The flux densities of the Gaussian and the point sources are $21.3 \pm 2.6$ and $14.3 \pm 1.0 \mathrm{Jy}$, respectively. The total flux density of the compact core (F1-4) is $35.6 \pm 3 \mathrm{Jy}$. 


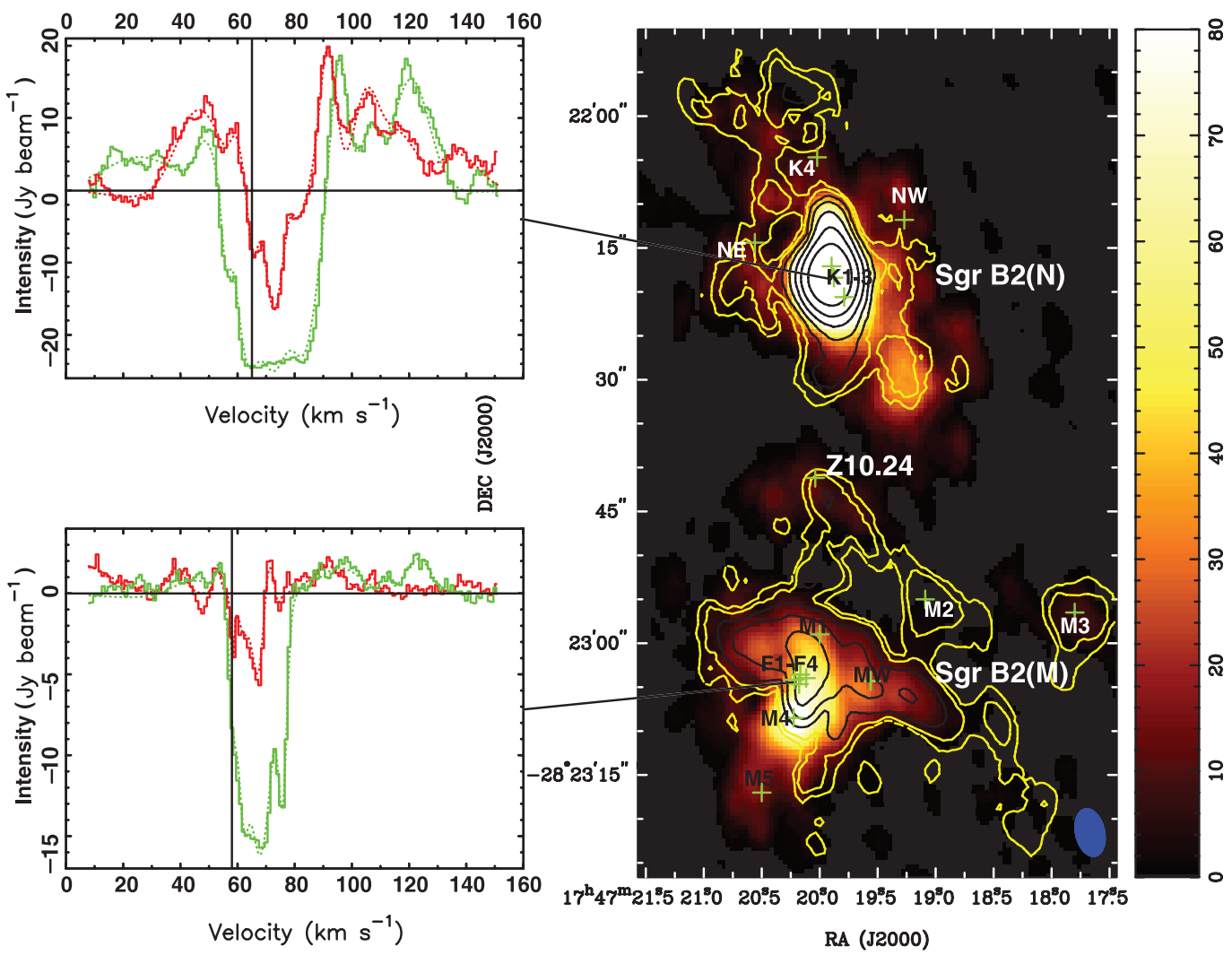

FIG. 3.- Mosaic images of integrated intensities with FWHM beam of $5.4^{\prime \prime} \times 3.2^{\prime \prime}$, P.A. $=12.5^{\circ}$. The pseudocolor is for integrated $\mathrm{H}_{2} \mathrm{CO}\left(3_{21}-2_{20}\right)$ emission intensity with $4 \sigma$ cutoff in each channel $\left(1 \sigma\right.$ is $\left.0.16 \mathrm{Jy}_{\text {beam }}^{-1}\right)$. The color scale on the right shows the range from 0 to $80 \mathrm{Jy} \mathrm{beam}^{-1} \mathrm{~km} \mathrm{~s}^{-1}$. The contours $(4,8,16,32,64,128$, $\left.256 \mathrm{Jy}_{\text {beam }}^{-1} \mathrm{~km} \mathrm{~s}^{-1}\right)$ are the integrated $\mathrm{H}_{2} \mathrm{CO}\left(3_{03}-2_{02}\right)$ emission intensity with $4 \sigma$ cutoff in each channel $\left(1 \sigma\right.$ is $0.18 \mathrm{Jy}$ beam $\left.{ }^{-1}\right)$. Top left: Observed spectra (solid curves) toward Sgr B2(N), green for $\mathrm{H}_{2} \mathrm{CO}\left(3_{03}-2_{02}\right)$ and red for $\mathrm{H}_{2} \mathrm{CO}\left(3_{21}-2_{20}\right)$. The dashed curves are the Gaussian fitting to the multiple components. Bottom left: Spectra toward Sgr B2(M). The vertical lines mark the systematic velocities, $58 \mathrm{~km} \mathrm{~s}^{-1}$ for Sgr B2(M) and $65 \mathrm{~km} \mathrm{~s}^{-1}$ for Sgr B2(N).

$I_{L} \approx I_{C} e^{-\tau_{L}}$. The optical depth $\left(\tau_{L}\right)$ can be derived from the formula

$$
\tau_{L}=-\ln \left(\frac{I_{L}}{I_{C}}\right)=-\ln \left(1+\frac{\Delta I_{L}}{I_{C}}\right),
$$

where $\Delta I_{L}=I_{L}-I_{C}$ is the observed line intensity and the $I_{C}$ is the observed continuum intensity. The errors in $\tau_{L}$ based on the fractional errors $\sigma_{I_{L}} / I_{L}$ of each line channel and $\sigma_{I_{C}} / I_{C}$ if the line is optically thin $\left[-\left(\Delta I_{L} / I_{C}\right)<1-e^{-1} \approx 0.6\right]$ are given by

$$
\sigma_{\tau} \approx \sqrt{\left(\sigma_{I_{L}} / I_{L}\right)^{2}+\left(\sigma_{I_{C}} / I_{C}\right)^{2}}
$$

In the optically thick case, the channels in the line center are saturated and the line-to-continuum ratio only gives a lower limit to the optical depth of the line. For example, if $n$ spectral channels are saturated by the absorption gas component at a velocity, we have $I_{L}<3 \sigma_{I_{L}} / \sqrt{n}$ in $3 \sigma$. Substituting this formula into the equation (1), the lower limit of the optical depth is

$$
\tau_{L}>-\ln \left(\frac{3 \sigma_{I_{L}}}{\sqrt{n} I_{C}}\right)
$$

With the Rayleigh-Jeans approximation, the value of $1 \mathrm{Jy} \mathrm{beam}^{-1}$ in our SMA observations is equivalent to $1.5 \mathrm{~K}$. The observed peak continuum intensities of the Sgr B2(N) and Sgr B2(M) cores are 29.2 and $20.2 \mathrm{Jy} \mathrm{beam}^{-1}$, which correspond to brightness temperatures of 44 and $31 \mathrm{~K}$ in our observations, respectively. The solution of radiation transfer function in terms of the observed brightness temperature of the line $\left(\Delta T_{L}^{\mathrm{obs}}\right)$ is given by

$$
\Delta T_{L}^{\mathrm{obs}}=\left(f_{L} T_{\mathrm{ex}}-f_{0} T_{C}^{\mathrm{obs}}\right)\left(1-e^{-\tau_{L}}\right),
$$

where $T_{\mathrm{ex}}$ is the excitation temperature of the molecular line and $T_{C}^{\mathrm{obs}}=f_{C} T_{C}$ is the observed brightness temperature of the continuum emission with the true brightness temperature of $T_{C} ; \tau_{L}$ is the optical depth of the molecular cloud; for given solid angles of the molecular cloud $\left(\Omega_{L}\right)$, continuum source $\left(\Omega_{C}\right)$, and the telescope beam $\left(\Omega_{B}\right), f_{L}=\Omega_{L} /\left(\Omega_{L}+\Omega_{B}\right)$ and $f_{C}=\Omega_{C} /\left(\Omega_{C}+\Omega_{B}\right)$ are the beam filling factors of the line and continuum, respectively, if both the source and the telescope beam are in Gaussian shape; $f_{0}$ denotes the fraction of the continuum source covered by the molecular cloud.

If the molecular cloud is in front of the larger continuum source $\left[f_{0} \leq\left(f_{L} / f_{C}\right)\right]$, the observed brightness temperature of the line $\left(T_{L}^{\mathrm{obs}}\right)$ becomes

$$
\Delta T_{L}^{\mathrm{obs}}=\left(f_{L} T_{\mathrm{ex}}-\frac{f_{L}}{f_{C}} T_{C}^{\mathrm{obs}}\right)\left(1-e^{-\tau_{L}}\right)
$$

If the molecular clouds cover the continuum cores with the same beam filling factor $\left(f_{L}=f_{C}\right)$, the upper limits of the excitation temperatures $\mathrm{H}_{2} \mathrm{CO}$ absorbing gas $\left(\Delta T_{L}^{\text {obs }}<0\right)$ are imposed by the observed continuum brightness temperatures of the continuum cores, i.e., $44 / f_{L}$ and $31 / f_{L} \mathrm{~K}$, for the Sgr B2(N) and Sgr B2(M) 
TABLE 2

The SMA Measurements of $\mathrm{H}_{2} \mathrm{CO}$ Lines

\begin{tabular}{|c|c|c|c|c|c|c|c|c|}
\hline \multirow[b]{2}{*}{ Source } & \multicolumn{3}{|c|}{$\mathrm{H}_{2} \mathrm{CO}\left(3_{03}-2_{02}\right)$} & \multirow[b]{2}{*}{$\tau_{1}$} & \multicolumn{3}{|c|}{$\mathrm{H}_{2} \mathrm{CO}\left(3_{21}-2_{20}\right)$} & \multirow[b]{2}{*}{$\tau_{2}$} \\
\hline & $\begin{array}{c}I_{\mathrm{p} 1} \\
\left(\mathrm{Jy} \mathrm{beam}^{-1}\right)\end{array}$ & $\begin{array}{c}V_{\mathrm{LSR} 1} \\
\left(\mathrm{~km} \mathrm{~s}^{-1}\right)\end{array}$ & $\begin{array}{c}\Delta V_{1} \\
\left(\mathrm{~km} \mathrm{~s}^{-1}\right)\end{array}$ & & $\begin{array}{c}I_{\mathrm{p} 2} \\
\left(\mathrm{Jy} \mathrm{beam}^{-1}\right)\end{array}$ & $\begin{array}{c}V_{\mathrm{LSR} 2} \\
\left(\mathrm{~km} \mathrm{~s}^{-1}\right)\end{array}$ & $\begin{array}{c}\Delta V_{2} \\
\left(\mathrm{~km} \mathrm{~s}^{-1}\right)\end{array}$ & \\
\hline \multirow[t]{8}{*}{ K1-3 …............................ } & $5.9 \pm 0.1$ & $49 \pm 0.5$ & $8 \pm 0.5$ & $\ldots$ & $11.3 \pm 0.2$ & $46 \pm 0.5$ & $18 \pm 0.5$ & $\ldots$ \\
\hline & $-8.3 \pm 0.3$ & $56 \pm 0.5$ & $3 \pm 0.5$ & $0.3 \pm 0.1$ & $4.7 \pm 0.1$ & $60 \pm 0.5$ & $5 \pm 0.5$ & $\ldots$ \\
\hline & $-19.0 \pm 1.6$ & $63 \pm 0.5$ & $9 \pm 0.5$ & $1.1 \pm 0.2$ & $-8.7 \pm 0.1$ & $65 \pm 0.5$ & $4 \pm 0.5$ & $0.4 \pm 0.1$ \\
\hline & $-23.5 \pm 0.7$ & $72 \pm 0.5$ & $12 \pm 1.2$ & $1.6 \pm 0.1$ & $-16.6 \pm 0.1$ & $73 \pm 0.5$ & $7 \pm 0.5$ & $0.8 \pm 0.1$ \\
\hline & $-20.0 \pm 1.1$ & $84 \pm 0.5$ & $11 \pm 0.5$ & $1.1 \pm 0.1$ & $-4.0 \pm 0.1$ & $82 \pm 0.5$ & $4 \pm 0.5$ & $0.1 \pm 0.1$ \\
\hline & $18.5 \pm 0.1$ & $96 \pm 0.5$ & $7 \pm 0.5$ & $\ldots$ & $19.6 \pm 0.1$ & $92 \pm 0.5$ & $7 \pm 0.5$ & $\ldots$ \\
\hline & $7.5 \pm 0.1$ & $106 \pm 0.5$ & $8 \pm 0.5$ & $\ldots$ & $12.1 \pm 0.5$ & $105 \pm 0.5$ & $10 \pm 0.5$ & $\ldots$ \\
\hline & $15.4 \pm 0.2$ & $121 \pm 0.5$ & $15 \pm 0.5$ & $\ldots$ & $7.8 \pm 0.2$ & $116 \pm 0.5$ & $15 \pm 0.9$ & $\ldots$ \\
\hline $\mathrm{K} 4 \ldots$ & $<0.54^{\mathrm{a}}$ & $\ldots$ & $\ldots$ & $\ldots$ & $<0.48^{\mathrm{a}}$ & $\ldots$ & $\ldots$ & $\ldots$ \\
\hline NE & $-1.2 \pm 0.1$ & $72 \pm 0.5$ & $20 \pm 1.1$ & $2.5 \pm 1.3$ & $-0.5 \pm 0.1$ & $71 \pm 0.9$ & $18 \pm 2.2$ & $0.5 \pm 0.2$ \\
\hline 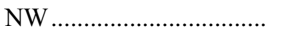 & $-1.1 \pm 0.1$ & $71 \pm 0.5$ & $18 \pm 1.3$ & $2.5 \pm 1.4$ & $<0.48^{\mathrm{a}}$ & $\ldots$ & $\ldots$ & $\ldots$ \\
\hline \multirow[t]{2}{*}{ 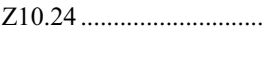 } & $-1.0 \pm 0.1$ & $70 \pm 1.1$ & $24 \pm 2.6$ & $0.7 \pm 0.1$ & $-0.3 \pm 0.1$ & $74 \pm 7.0$ & $15 \pm 9.5$ & $0.2 \pm 0.1$ \\
\hline & $1.2 \pm 0.1$ & $90 \pm 0.5$ & $11 \pm 1.1$ & $\ldots$ & $0.3 \pm 0.1$ & $87 \pm 6.5$ & $13 \pm 8.5$ & $\ldots$ \\
\hline \multirow[t]{8}{*}{ F1-4 …............................. } & $0.9 \pm 0.7$ & $45 \pm 2.0$ & $18 \pm 7.8$ & $\ldots$ & $1.6 \pm 0.1$ & $39 \pm 0.5$ & $7 \pm 0.5$ & $\ldots$ \\
\hline & $1.6 \pm 0.2$ & $54 \pm 0.5$ & $2 \pm 0.5$ & $\ldots$ & $1.5 \pm 0.1$ & $53 \pm 0.5$ & $3 \pm 0.5$ & $\ldots$ \\
\hline & $-6.1 \pm 0.4$ & $58 \pm 0.5$ & $3 \pm 0.5$ & $0.4 \pm 0.1$ & $-3.8 \pm 0.3$ & $58 \pm 0.5$ & $2 \pm 0.5$ & $0.2 \pm 0.1$ \\
\hline & $-7.6 \pm 0.7$ & $62 \pm 0.5$ & $4 \pm 0.5$ & $0.5 \pm 0.1$ & $-2.2 \pm 0.4$ & $62 \pm 0.8$ & $4 \pm 1.4$ & $0.1 \pm 0.1$ \\
\hline & $-16 \pm 0.1$ & $68 \pm 0.5$ & $10 \pm 0.5$ & $1.6 \pm 0.1$ & $-5.1 \pm 0.9$ & $67 \pm 1.5$ & $5 \pm 3.4$ & $0.3 \pm 0.1$ \\
\hline & $-10 \pm 0.3$ & $76 \pm 0.5$ & $3 \pm 0.5$ & $0.7 \pm 0.1$ & $-2.0 \pm 0.4$ & $74 \pm 0.6$ & $2 \pm 1.0$ & $0.1 \pm 0.1$ \\
\hline & $1.6 \pm 0.1$ & $96 \pm 0.5$ & $17 \pm 0.9$ & $\ldots$ & $1.7 \pm 0.1$ & $91 \pm 0.5$ & $13 \pm 0.6$ & $\ldots$ \\
\hline & $1.1 \pm 0.1$ & $112 \pm 0.5$ & $3 \pm 0.5$ & $\ldots$ & $1.0 \pm 0.1$ & $107 \pm 0.5$ & $5 \pm 0.6$ & $\ldots$ \\
\hline ME ............................... & $<0.76^{\mathrm{a}}$ & $\ldots$ & $\ldots$ & $\ldots$ & $<0.67^{\mathrm{a}}$ & $\ldots$ & $\ldots$ & $\ldots$ \\
\hline \multirow[t]{2}{*}{ 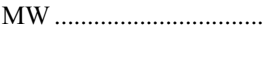 } & $6.6 \pm 0.1$ & $53 \pm 0.5$ & $6 \pm 0.5$ & $\cdots$ & $4.3 \pm 0.1$ & $53 \pm 0.5$ & $6 \pm 0.5$ & $\cdots$ \\
\hline & $-0.7 \pm 0.1$ & $70 \pm 0.9$ & $19 \pm 2.4$ & $0.5 \pm 0.1$ & $-0.1 \pm 0.1$ & $70 \pm 3.8$ & $17 \pm 9.8$ & $0.1 \pm 0.1$ \\
\hline \multirow[t]{2}{*}{ 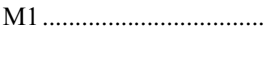 } & $6.7 \pm 0.4$ & $52 \pm 0.5$ & $11 \pm 0.5$ & $\ldots$ & $4.0 \pm 0.1$ & $52 \pm 0.5$ & $11 \pm 0.5$ & $\ldots$ \\
\hline & $-1.4 \pm 0.1$ & $64 \pm 2.3$ & $21 \pm 3.4$ & $0.9 \pm 0.1$ & $-1.0 \pm 0.1$ & $64 \pm 1.2$ & $12 \pm 2.1$ & $0.6 \pm 0.1$ \\
\hline M2.. & $2.6 \pm 0.1$ & $55 \pm 0.5$ & $6 \pm 0.5$ & $\ldots$ & $0.5 \pm 0.1$ & $53 \pm 0.5$ & $4 \pm 1.1$ & $\ldots$ \\
\hline 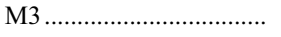 & $1.9 \pm 0.1$ & $53 \pm 0.5$ & $7 \pm 0.5$ & $\ldots$ & $0.8 \pm 0.1$ & $55 \pm 0.5$ & $9 \pm 0.9$ & $\cdots$ \\
\hline \multirow[t]{5}{*}{ M4 f............................. } & $-2.1 \pm 0.1$ & $51 \pm 0.5$ & $7 \pm 0.5$ & $0.8 \pm 0.1$ & $<0.48^{\mathrm{a}}$ & $\ldots$ & $\ldots$ & $\cdots$ \\
\hline & $-2.2 \pm 0.1$ & $65 \pm 0.7$ & $14 \pm 1.5$ & $0.9 \pm 0.1$ & $5.9 \pm 0.1$ & $66 \pm 0.5$ & $10 \pm 0.5$ & $\ldots$ \\
\hline & $-0.8 \pm 0.3$ & $70 \pm 0.5$ & $3 \pm 1.1$ & $0.2 \pm 0.1$ & $4.2 \pm 0.2$ & $70 \pm 0.5$ & $3 \pm 0.5$ & $\cdots$ \\
\hline & $-1.6 \pm 0.3$ & $75 \pm 0.5$ & $3 \pm 0.6$ & $0.5 \pm 0.1$ & $<0.48^{\mathrm{a}}$ & $\ldots$ & $\ldots$ & $\ldots$ \\
\hline & $1.8 \pm 0.1$ & $100 \pm 0.5$ & $8 \pm 0.5$ & $\ldots$ & $<0.48^{\mathrm{a}}$ & $\ldots$ & $\ldots$ & $\ldots$ \\
\hline 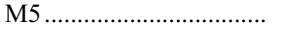 & $0.2 \pm 0.1$ & $66 \pm 3.1$ & $16 \pm 7.4$ & $\cdots$ & $1.2 \pm 0.1$ & $64 \pm 0.5$ & $14 \pm 0.8$ & $\cdots$ \\
\hline
\end{tabular}

a $3 \sigma$ limit.

cores. For a given filling factor $f_{L}=0.3$, the excitation temperatures of $\mathrm{H}_{2} \mathrm{CO}$ would be less than 150 and $103 \mathrm{~K}$ for Sgr B2(N) and Sgr B2(M), respectively.

\subsubsection{Individual Components}

Figure 4 shows the spectra of the $\mathrm{H}_{2} \mathrm{CO}$ lines (averaged over one beam) made from the image cube for the rest of components. Each of spectral panels includes both the lower $\left(3_{03}-2_{02}\right.$, the green profile) and the higher $\left(3_{21}-2_{20}\right.$, the red profile) transition lines. Gaussian fits to the $\mathrm{H}_{2} \mathrm{CO}$ spectra were carried out for both absorption and emission components. The emission and absorption probably come from the different regions along the line of sight. The angular resolution of our observations appears to be inadequate to distinguish the discrete components in the cores. However, our high spectral resolution is adequate to separate the emission from the absorption in the Gaussian fits. The parameters derived from the Gaussian fits to the two $\mathrm{H}_{2} \mathrm{CO}$ transitions are summarized in Table 2 , including the central line velocity $\left(V_{\mathrm{LSR}}\right)$, the full width at half-maximum $(\Delta V)$, and peak intensity $\left(I_{p}\right)$.

The M1, M4, and M5 are located along the major axis of the bipolar outflow originated from the F cluster (Lis et al. 1993). M1 is on the blueshifted side of the outflow. Both the high- and low-transition spectra can be fitted with two Gaussian compo- nents at $52 \mathrm{~km} \mathrm{~s}^{-1}$ in emission and $64 \mathrm{~km} \mathrm{~s}^{-1}$ in absorption. The weak redshifted absorption with respect to the systematic velocity suggests that a relatively cold gas component in front of the continuum source is moving toward it. The strong blueshifted emission with respect to the systematic velocity is the highly excited gas emission in the outflow (in front of the continuum source), likely mixed with the infall gas emission (behind the continuum source).

Sgr B2(M)-M4 is located close to the $\mathrm{H}$ II regions, i.e., the F1-4 cluster. The higher transition spectrum can be fitted with two Gaussian components in emission at 66 and $70 \mathrm{~km} \mathrm{~s}^{-1}$, both of which are redshifted with respect to the systematic velocity $58 \mathrm{~km} \mathrm{~s}^{-1}$. The lower transition spectrum can be fitted with five Gaussian components at velocity $51,65,70,75 \mathrm{~km} \mathrm{~s}^{-1}$ in absorption and $100 \mathrm{~km} \mathrm{~s}^{-1}$ in emission. The morphology of this region is a complex.

A possible model to interpret the spectral characteristics of M4 is considered here. If we assume a non-LTE condition for the gas and that both higher and lower transition gas comes from the same gas clump located in front of the continuum core, the excitation temperature of the higher transition gas is larger than the brightness temperature of the continuum, while the excitation temperature of the lower transition gas is less than the brightness temperature 

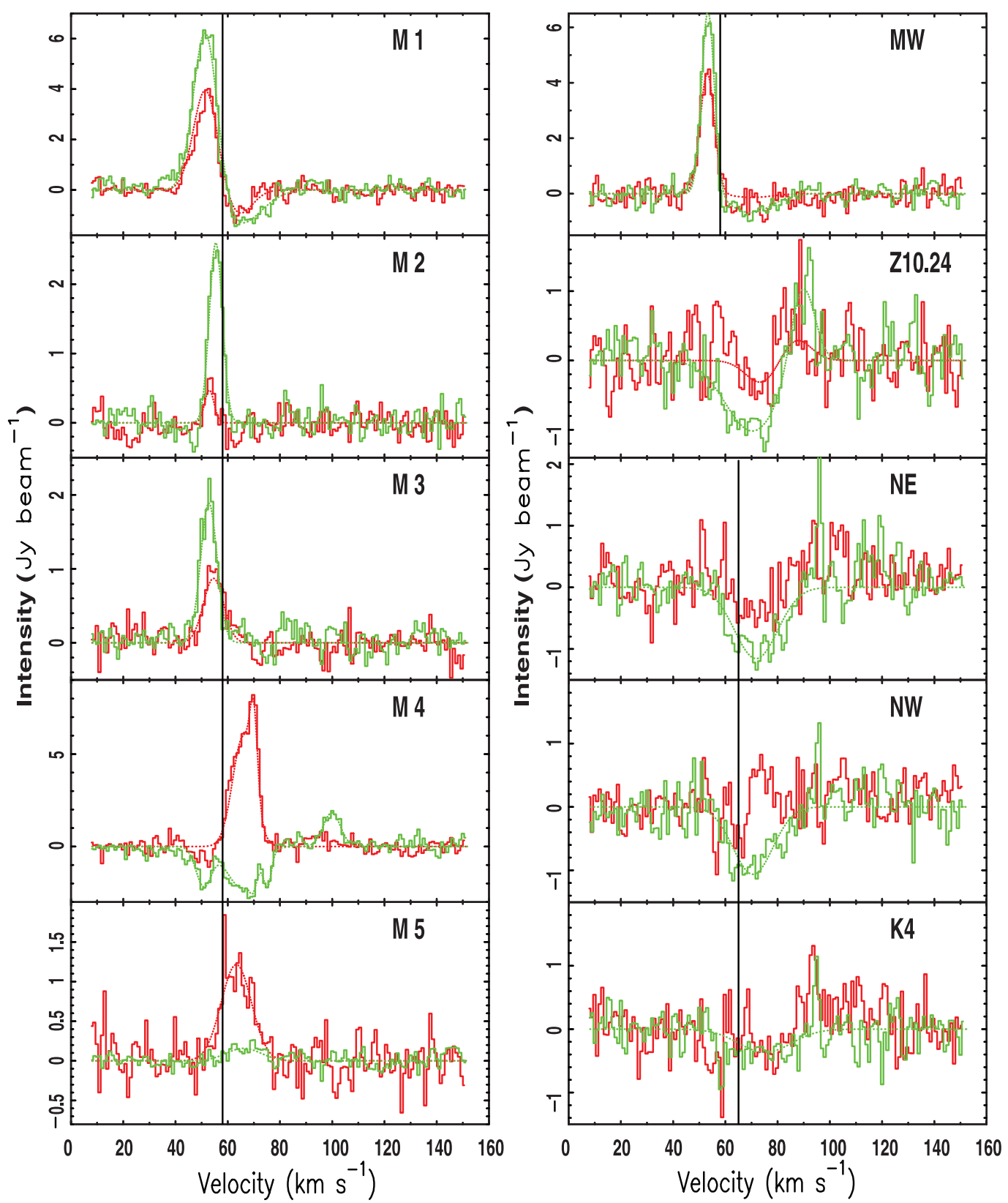

FIG. 4. - Spectra from various components, green for $\mathrm{H}_{2} \mathrm{CO}\left(3_{03}-2_{02}\right)$ and red for $\mathrm{H}_{2} \mathrm{CO}\left(3_{21}-2_{20}\right)$. Solid curves are the observed spectra and dashed curves are the multiple Gaussian fitting. The vertical lines mark the systematic velocities, $58 \mathrm{~km} \mathrm{~s}^{-1}$ for M1, M2, M3, M4, M5, and MW in Sgr B2(M) and 65 km s${ }^{-1}$ for NE, NW, and K4 in Sgr B2(N).

of the continuum. The redshifted absorption suggests that the gas flows toward the continuum core. The nature of absorption and emission at M4 suggests that the process of excitation of the infall molecular cloud is complicated. The radiative excitation by the strong FIR radiation field might play an important role in the region near the core since the collision alone cannot produce the observed line ratio or the inverse population between the higher transition in $K_{-1}=2$ and the lower transition in $K_{-1}=0$ based on the LVG fitting (see Appendix B).

The inversion in population between the two lowest $\mathrm{K}$ ladders is observed best in the redshifted outflow component. At M5, the spectrum of the higher transition gas shows a very significant line emission (7-8 $\sigma)$, fitted to a Gaussian at $66 \mathrm{~km} \mathrm{~s}^{-1}$ with a line width of $16 \mathrm{~km} \mathrm{~s}^{-1}$, while less significant emission $(1-2 \sigma)$ of the lower transition gas is shown at the same position. The highly reversed line ratio $I\left(3_{21}-2_{20}\right) / I\left(3_{03}-2_{02}\right) \sim 6$ suggests that local thermodynamic equilibrium (LTE) is not valid at this location and a weak maser process is active in the outflow region.

The gas components M2 and M3 and MW show significant line emission $(>10 \sigma)$ from the lower transition gas while the higher transition emission is relatively weak. The line ratio $I\left(3_{03}-2_{02}\right) / I\left(3_{21}-2_{20}\right)$ in those isolated components varies in the range between 1.5 and 5.2.

$\mathrm{H}_{2} \mathrm{CO}$ maser at $6 \mathrm{~cm}$ (Mehringer et al. 1994) was detected in the Z10.24 region. The SMA spectrum of the lower transition at $\mathrm{Z} 10.24$ shows that a significant amount of gas is in absorption while the emission is present but redshifted with respect to the absorption feature, a typical P Cygni profile suggesting an outflow in this region. The spectrum of the higher $\mathrm{H}_{2} \mathrm{CO}$ transition can be fitted with two emission Gaussian components at 50 and 


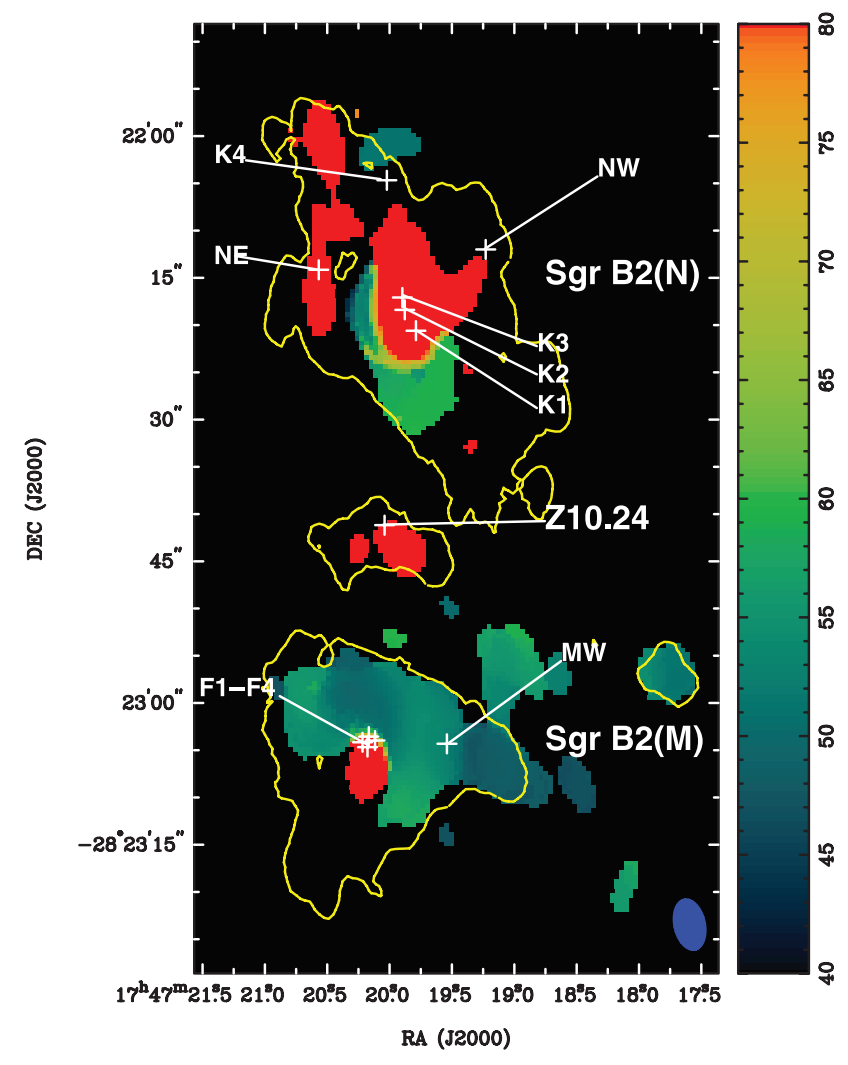

FIG. 5.- Mosaic image of the intensity-weighted velocity map in pseudocolor constructed from the data after imposing a cutoff of $8 \sigma$, showing velocity field traced by $\mathrm{H}_{2} \mathrm{CO}\left(3_{03}-2_{02}\right)$ emission in pseudocolor. The scale on the right shows the velocity range from 40 to $80 \mathrm{~km} \mathrm{~s}^{-1}$. The contours outline the integrated intensity of $\mathrm{H}_{2} \mathrm{CO}\left(3_{21}-2_{20}\right)$ emission at the level of $4 \mathrm{Jy}$ beam ${ }^{-1} \mathrm{~km} \mathrm{~s}^{-1}$. The FWHM beam is $5.4^{\prime \prime} \times 3.2^{\prime \prime}\left(\right.$ P.A. $\left.=12.5^{\circ}\right)$.

$84 \mathrm{~km} \mathrm{~s}^{-1}$ with an absorbing Gaussian at $75 \mathrm{~km} \mathrm{~s}^{-1}$, which is consistent with the H66 $\alpha$ transition (de Pree et al. 1996). Excluding the possibility of the expanding shell model, de Pree et al. argued that an ionized outflow is likely centered at $\mathrm{Z10.24}$. Our $\mathrm{H}_{2} \mathrm{CO}$ observations appear to favor their argument of a bipolar outflow from the UCH II region.

Toward the NE continuum source, both the higher and lower $\mathrm{H}_{2} \mathrm{CO}$ transitions show that the majority of the gas in absorption is redshifted with respect to the mean systematic velocity $65 \mathrm{~km} \mathrm{~s}^{-1}$.

Toward NW, a broad $\left(\Delta V=18 \pm 1.3 \mathrm{~km} \mathrm{~s}^{-1}\right)$ absorption $\left(-1.1 \mathrm{Jy} \mathrm{beam}^{-1}\right)$ from the lower transition line is detected at $71 \mathrm{~km} \mathrm{~s}^{-1}$. The spectrum of the higher transition shows no significant lines in either emission or absorption.

\subsection{Kinematics}

Figures 5 and 6 show the images of intensity-weighted velocity (or moment 1) of the $\mathrm{H}_{2} \mathrm{CO}$ emission gas from both transitions. The moment 1 maps were constructed with a cutoff of $8 \sigma$ from each of the channel images in the velocity range of $8-151 \mathrm{~km} \mathrm{~s}^{-1}$.

In the Sgr B2(M) region, the kinematical structure observed from the lower transition (see Fig. 5) consists of the highly redshifted components $5^{\prime \prime}$ southeast of the compact core and a northeast-southwest arch structure. The component M4 appears to be a fast moving compact component $\left(V_{\mathrm{LSR}} \sim 100 \mathrm{~km} \mathrm{~s}^{-1}\right)$ ejected from the core. The morphology of the northeast-southwest arch in Sgr B2(M) from the velocity field in the higher transition gas (see Fig. 6) appears to be consistent with that observed in the lower transitions. A velocity gradient is present southeast of

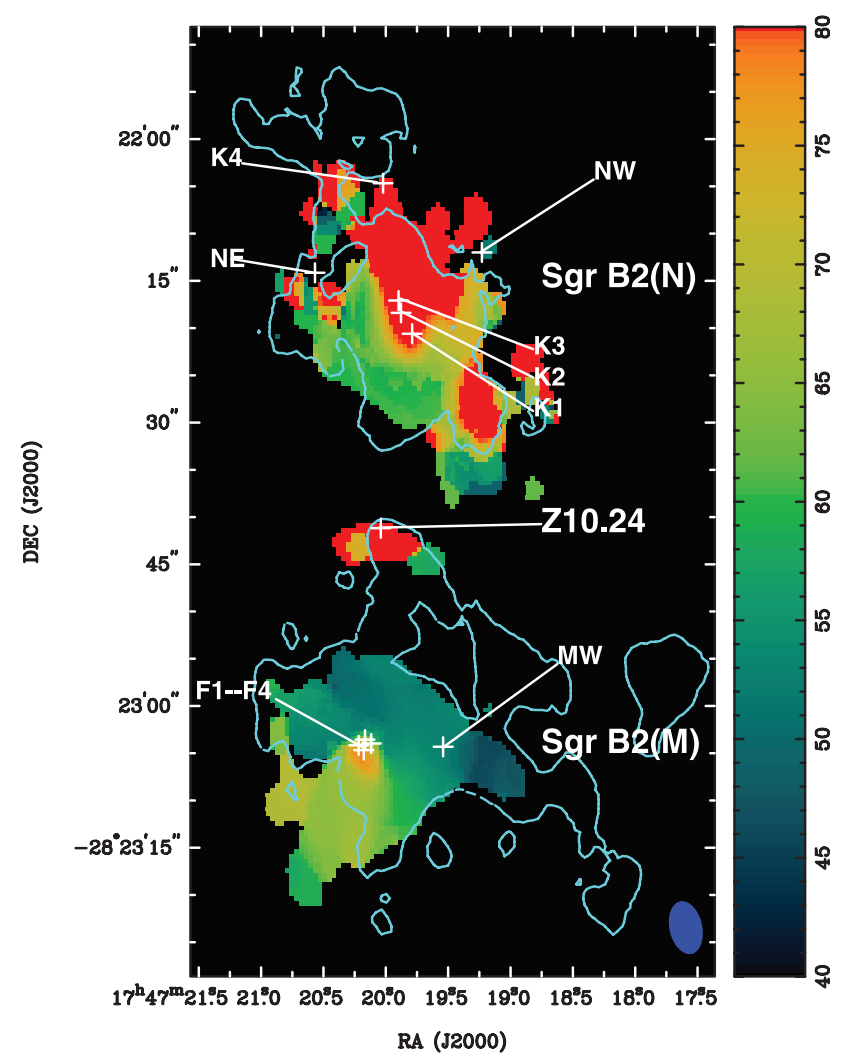

FIG. 6.-Mosaic image of the intensity-weighted velocity map in pseudocolor constructed from the data after imposing a cutoff of $8 \sigma$, showing velocity field traced by $\mathrm{H}_{2} \mathrm{CO}\left(3_{21}-2_{20}\right)$ emission in pseudocolor. The scale shows the velocity range from 40 to $80 \mathrm{~km} \mathrm{~s}^{-1}$. The contours outline the integrated intensity of $\mathrm{H}_{2} \mathrm{CO}\left(3_{03}-2_{02}\right)$ emission at the level of $4 \mathrm{Jy}_{\text {beam }}{ }^{-1} \mathrm{~km} \mathrm{~s}^{-1}$. The FWHM beam is $5.4^{\prime \prime} \times 3.2^{\prime \prime}\left(\right.$ P.A. $\left.=12.5^{\circ}\right)$.

M4 (shown in Fig. 6), which appears to indicate a decelerating outflow. In the case of Sgr B2(M), the higher $\mathrm{H}_{2} \mathrm{CO}$ transition appears to trace outflow well, which is consistent with the interpretation of the larger scale mass outflow based on the lower angular-resolution observations of $\mathrm{NH}_{3}$ and $\mathrm{SO}$ (Vogel et al. 1987). The higher angular-resolution observations of $\mathrm{NH}_{3}$ (Guame \& Claussen 1990) showed that the redshifted emission is located south of the F3 H II region, and the blueshifted absorption is located north of the redshifted emission, showing a velocity gradient in the north-south direction. Based on their higher angularresolution observations of $\mathrm{OH}$ maser and $\mathrm{NH}_{3}$, Guame \& Claussen (1990) suggested that the north-south velocity gradient can be explained by a rotating disk or a torus of material with an extent of $2.5^{\prime \prime}$ surrounding the Sgr B2(M)-F cluster. Although the angular resolution in our observations is not adequate to verify the kinematical model proposed by Guame \& Claussen (1990), our observed arch-like morphology of the $\mathrm{H}_{2} \mathrm{CO}$ gas shown in blueshifted emission from northeast to southwest in the larger scale also indicates that the gas is undergoing a complicated infall process interacting with the outflow while the gas is spiraling onto the core rather than being in simple free fall.

In the Sgr B2(N) region, the lower transition map (see Fig. 5) shows the redshifted velocity located north of K2 and blueshifted velocity located south of $\mathrm{K} 2$ with a north-south velocity gradient across $\mathrm{K} 2$. A similar velocity gradient has been observed in the $\mathrm{HC}_{3} \mathrm{~N}$ line (Lis et al. 1993). These authors argued that the northsouth velocity gradient in $\mathrm{Sgr} \mathrm{B} 2(\mathrm{~N})$ traces rotation. The kinematics of the lower $\mathrm{H}_{2} \mathrm{CO}$ transition in emission gas of $\mathrm{Sgr} \mathrm{B} 2(\mathrm{~N})$ 


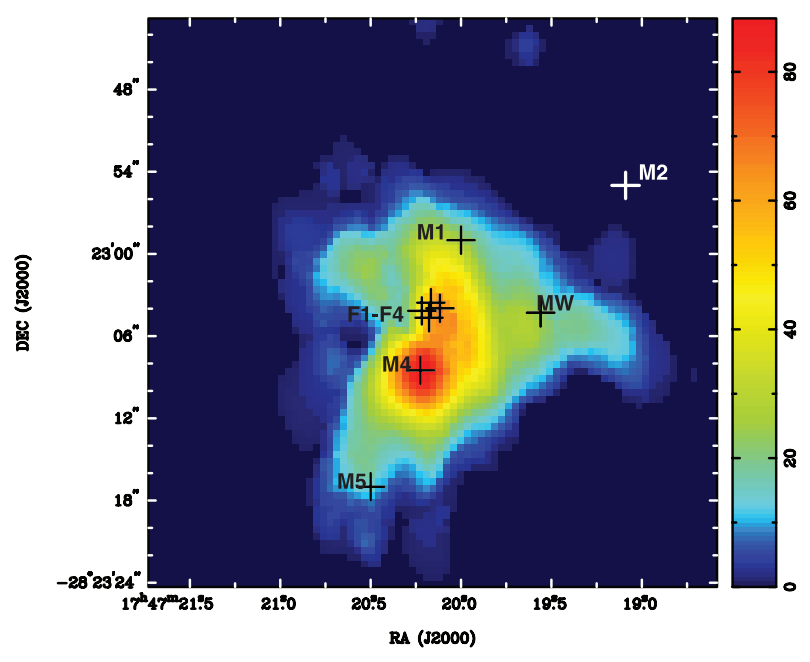

FIG. 7.- Integrated line intensity of $\mathrm{H}_{2} \mathrm{CO}\left(3_{21}-2_{20}\right)$ in Sgr B2(M), showing the emission from the decelerating outflow along the direction in P.A. $=158^{\circ}$. The color scale gives the intensity from 0 to $90 \mathrm{Jy} \mathrm{beam}^{-1} \mathrm{~km} \mathrm{~s}^{-1}$. The FWHM beam is $5.4^{\prime \prime} \times 3.2^{\prime \prime}\left(\right.$ P.A. $\left.=12.5^{\circ}\right)$.

is likely dominated by the gas rotating around the core. In addition to the north-south velocity gradient from the higher transition $\mathrm{H}_{2} \mathrm{CO}\left(3_{21}-2_{20}\right)$ map as observed in the lower transition gas, an east-west velocity gradient is also observed in Sgr B2(N) (see Fig. 6). The outflow in Sgr B2(N) was observed in the east-west direction (Lis et al. 1993). The higher angular-resolution observations of $\mathrm{NH}_{3}$ (Gaume \& Claussen 1990) appeared to show a southeast-northwest velocity gradient across K2. The southeastnorthwest velocity gradient in the larger scale from our observations appears to be consistent with the kinematical structure observed in $\mathrm{NH}_{3}$. Based on the higher angular-resolution observations of $\mathrm{NH}_{3}$, ruling out a simple outflow/rotation model, Gaume \& Claussen (1990) suggested that several kinematic components in outflow, infall, and rotation might be involved in the Sgr B2(N) core. The southeast-northwest velocity gradient observed from the $\mathrm{H}_{2} \mathrm{CO}$ emission gas in $\mathrm{Sgr} \mathrm{B} 2(\mathrm{~N})$ appears to be caused by a combination of rotation, infall, and outflow. The angular resolution of our observations is not adequate to discern the details of these motions.

\section{OUTFLOW AND INFALL}

In order to better understand the ongoing astrophysical processes in the star formation cores, we modeled the observed kinematics and the ratio of the line intensities. In comparison to Sgr B2(N), the kinematics observed from Sgr B2(M) appears to be relatively simple and characterized by infall and outflow. Figure 7 shows the single field map of $\mathrm{H}_{2} \mathrm{CO}\left(3_{21}-2_{20}\right)$ centered at the $\mathrm{F}$ cluster. The major axis of the redshifted outflow (P.A. $=$ $158^{\circ}$ ) can be drawn by connecting the continuum core and the tips of the outflows (M1 and M5).

Along the major axis of outflow (P.A. $=158^{\circ}$ ), we have made a position-velocity diagram (PV) from the higher $\mathrm{H}_{2} \mathrm{CO}$ transition line cube (see Fig. 8). The solid contours show the emission and dashed contours indicate the absorption. This diagram shows two distinct decelerating outflow components clearly. The redshifted component shows that the emission near the core (position at $0^{\prime \prime}$ in the vertical axis) starts with a high velocity of $106 \mathrm{~km} \mathrm{~s}^{-1}$ and the velocity declines to a terminal velocity at $58 \mathrm{~km} \mathrm{~s}^{-1}$ as the gas goes to the outer region (20"away from the core). If we shifted velocity to the terminal velocity $58 \mathrm{~km} \mathrm{~s}^{-1}$ or

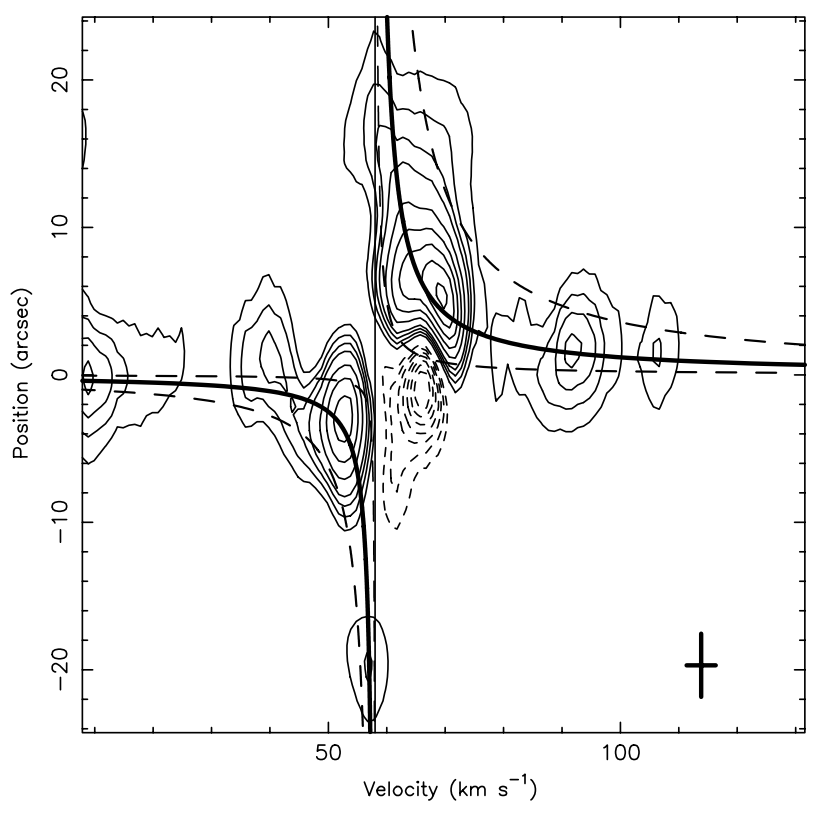

FIG. 8.-Velocity-position diagram cutting along the major axis of the outflows (see Fig. 7), which was constructed from the $\mathrm{H}_{2} \mathrm{CO}\left(3_{21}-2_{20}\right)$ spectral line cube. The solid contours show the emission from the decelerating outflows. The dashed contours are the absorption. The effective resolution of this map is labeled with the plus sign at bottom right corner, i.e., $5 \mathrm{~km} \mathrm{~s}^{-1}$ in velocity and $5^{\prime \prime}$ in position. The vertical green line marks the systematic velocity. The solid curves are the best fitting to the decelerating outflow, with the decelerating outflow parameter $V_{0} r_{\min } f(\Theta, \varphi)=2 \mathrm{~km} \mathrm{~s}^{-1}$ pc for the redshifted component and $V_{0} r_{\min } f(\Theta, \varphi)=0.8 \mathrm{~km} \mathrm{~s}^{-1} \mathrm{pc}$ for the blueshifted. The dashed color lines show the range of $V_{0} r_{\min } f(\Theta, \varphi)$ between 0.4 and $6 \mathrm{~km} \mathrm{~s}^{-1} \mathrm{pc}$ for the redshifted component and 0.1 to $2 \mathrm{~km} \mathrm{~s}^{-1}$ pc for the blueshifted component. [See the electronic edition of the Journal for a color version of this figure.]

in the system rest frame, the decelerating redshifted outflow is mainly located in the first quadrant with the absolute velocity decreasing from $\sim 48$ to $0 \mathrm{~km} \mathrm{~s}^{-1}$. The third quadrant shows the decelerating blueshifted outflow component with deceleration from $\sim 50$ to $0 \mathrm{~km} \mathrm{~s}^{-1}$.

Appendix A describes the numerical calculations that have been carried out to model the kinematical characteristics of a decelerating outflow combined with spherical infall. With a simple assumption of mass conservation and power-law distributions in both outflow velocity and molecular density, the observed kinematics of the outflow components can be well fitted to the decelerating outflow model with an initial velocity of $V_{0}=83 \mathrm{~km} \mathrm{~s}^{-1}$ at $r_{0}=$ $1^{\prime \prime}(0.04 \mathrm{pc})$ from the core center with an outflow opening angle of $\Theta=30^{\circ}$ and inclination of $\varphi=45^{\circ}$ for the redshifted component (the thick curve to the upper right in Fig. 8). The blueshifted component can be fitted with $V_{0}=33 \mathrm{~km} \mathrm{~s}^{-1}$ at $r_{0}=1^{\prime \prime}$ ( $0.04 \mathrm{pc}), \Theta=30^{\circ}$, and $\varphi=45^{\circ}$ (thick blue curve). The dashed curves range initial velocity $V_{0}$ at $r_{0}=1^{\prime \prime}$ given the same opening angle and inclination angle. For the redshifted outflow, $V_{0}$ is from 250 to $17 \mathrm{~km} \mathrm{~s}^{-1}$. The blueshifted velocity ranges from 83 to $4 \mathrm{~km} \mathrm{~s}^{-1}$.

Our observations and analysis have shown the presence of a decelerating outflow from the core in Sgr B2(M). Such a decelerating outflow can be caused by the entrainment of gas through the interaction between a fast wind flow from the central stars and an ambient cloud core, where the incorporation of additional mass into the outflow decelerates the wind (Lizano et al. 1988). As the wind moves farther from the cores, the outflow velocity terminates at the systematic velocity $58 \mathrm{~km} \mathrm{~s}^{-1}$ of the molecular cloud. We note that both the redshifted and blueshifted outflows 
terminate at a common velocity, which is close to the mean systematic velocity $61 \mathrm{~km} \mathrm{~s}^{-1}$ of Sgr B2(M) (Sutton et al. 1991). In fact, the common terminal velocity $58 \pm 2 \mathrm{~km} \mathrm{~s}^{-1}$ of blueshifted and redshifted outflow appears to provide an unambiguous method, independent of the chemical processes in molecular clouds, for accurately measuring the systematic velocity of $\operatorname{Sgr} B 2(M)$. The uncertainty in the terminal velocity is mainly due to the velocity resolution in the PV diagram and the uncertainty in the model fitting process.

Finally, the absorption located in the second quadrant is shown to be redshifted with respect to the systematic velocity $58 \pm 2 \mathrm{~km} \mathrm{~s}^{-1}$. Thus, with the accurately determined systematic velocity we can be certain that this absorption feature arises from gas flowing toward the continuum source.

\section{EXCITATION}

Based on the line ratio of the two transitions from the spectra, most of the gas in the Sgr B2 region does not satisfy the LTE condition. Under the assumption that the molecular cloud has spherical geometry, the non-LTE calculation requiring the collisional excitation rates for $\mathrm{H}_{2} \mathrm{CO}$ (Green 1991) has been carried out for Sgr B2 using the large velocity gradient (LVG) approximation (e.g., Evans et al. 1979; Scoville \& Solomon 1974; Lucy 1971). The radiative transfer calculations and model fitting are discussed in details in Appendix B. The fitting results for the line components for models with various sets of parameters are given in Table 3.

We summarize the excitation properties for the various $\mathrm{H}_{2} \mathrm{CO}$ components in the following:

1. Toward the absorption components of the cores (K1-3, F1-4), the constraint on the kinetic temperature depends on the selection of the beam filling factor of the continuum emission, ranging from a few tens of $\mathrm{K}$ for a large continuum beam $f_{C}(0.3)$ to a few hundred $\mathrm{K}$ for a small $f_{C}(0.05)$. High angular resolution observations are necessary to precisely determine the range of $T_{k}$. The typical values of $n_{\mathrm{H}_{2}}$ are $10^{5}-10^{7} \mathrm{~cm}^{-3}$ for the core components. The derived column den sities $N_{\text {para- } \mathrm{H}_{2} \mathrm{CO}} / \Delta V$ are in the range of $10^{14}-10^{17} \mathrm{~cm}^{-2} \mathrm{~km}^{-1} \mathrm{~s}$.

2. For the discrete $\mathrm{H}_{2} \mathrm{CO}$ components, no LVG solutions could be fitted to the observations with either model A or model $\mathrm{B}$ in which $f_{C}$ is large and the brightness temperature of the background radiation is too small to produce significant absorption lines as observed. For excitation temperature of a few tens of $\mathrm{K}$ and higher, the intensity of absorption (see eq. [5]) suggests that the beam filling factor must be small, i.e., $f_{C} \sim 0.1$ or smaller, for a region with a continuum peak intensity of $\sim 2 \mathrm{Jy} \mathrm{beam}^{-1}$ in our observations. The kinetic temperatures derived from the LVG fitting are all below $100 \mathrm{~K}$ for both emission and absorption components except for M3 emission component in which $T_{k}=$ $150_{-50}^{+250} \mathrm{~K}$. In general the components outside the cores are cooler. The typical values of $n_{\mathrm{H}_{2}}$ are $10^{4}-10^{6} \mathrm{~cm}^{-3}$ for the discrete components. The derived column density $N_{\text {para- } \mathrm{H}_{2} \mathrm{CO}} / \Delta V$ is in the range of $10^{13}-10^{16} \mathrm{~cm}^{-2} \mathrm{~km}^{-1} \mathrm{~s}$.

3. For the components in the redshifted outflow of Sgr B2(M), the line ratio $I\left(3_{03}-2_{02}\right) / I\left(3_{21}-2_{20}\right)$ is significantly smaller than unity, suggesting that the population of the ground levels in the two K ladders $\left(K_{-1}=0\right.$ and 2$)$ is inverted. Hence, a weak maser process between the two different $\mathrm{K}$ ladders must occur in this region. Based on our radiative transfer code with the collisional excitation rates for kinetic temperature $T_{k} \leq 300 \mathrm{~K}$ (Green 1991), we cannot rule out the possibility that the $\mathrm{H}_{2} \mathrm{CO}\left(3_{21}-2_{20}\right)$ line in Sgr B2(M) outflow is excited in the high temperature $\mathrm{C}$ shocks. On the other hand, the strong FIR radiation field near the Sgr B2(M) core might also play a role in the population inversion of the molecule.

\section{DISCUSSION}

The assessment of molecular cloud mass from molecular lines can be affected by the excitation, opacity, abundance variations, and gas dynamics of the molecular lines. The optically thin submillmeter dust continuum emission has been proven to be a good tracer of molecular cloud mass (Pierce-Price et al. 2000; Gordon 1995). If we take an average grain radius of $0.1 \mu \mathrm{m}$ and grain density of $3 \mathrm{~g} \mathrm{~cm}^{-3}$ and a gas to dust ratio of 100 (Hildebrand 1983; Lis et al. 1991), the dusty cloud mass and column density are given by the formulae (Lis et al. 1991)

$$
\begin{gathered}
M_{\mathrm{H}_{2}}=1.3 \times 10^{4} \frac{e^{h \nu / \kappa T}-1}{Q(\nu)}\left(\frac{S_{\nu}}{\mathrm{Jy}}\right)\left(\frac{D}{\mathrm{kpc}}\right)^{2}\left(\frac{\nu}{\mathrm{GHz}}\right)^{-3} M_{\odot}, \\
N_{\mathrm{H}_{2}}=8.1 \times 10^{17} \frac{e^{h \nu / \kappa T}-1}{Q(\nu) \Omega}\left(\frac{S_{\nu}}{\mathrm{Jy}}\right)\left(\frac{\nu}{\mathrm{GHz}}\right)^{-3}\left(\mathrm{~cm}^{-2}\right),
\end{gathered}
$$

where $T$ is the mean dust temperature $(\mathrm{K}), Q(\nu)$ is grain emissivity at frequency $\nu, S_{\nu}$ is the flux density corrected for free-free emission, and $\Omega$ is the solid angle subtended by the source. Assuming $Q(\nu)$ at $1.3 \mathrm{~mm}$ is $2 \times 10^{-5}$ and the dust temperature is 150 K for Sgr B2 (Carlstrom \& Vogel 1989; Lis et al. 1993; Kuan et al. 1996), we derived the masses, $\mathrm{H}_{2}$ column densities, and number densities.

Because the continuum at $1.3 \mathrm{~mm}$ contains free-free emission, we estimate the physical parameters using the flux densities of the continuum corrected for free-free emission. Assuming $3.6 \mathrm{~cm}$ continuum emission with FWHM beam of $3.8^{\prime \prime} \times 2.0^{\prime \prime}$ (Mehringer et al. 1993) of Sgr B2(N) (K1-3) and Sgr B2(M) (F1-4) come from optically thin free-free emission $\left(S_{\nu} \propto \nu^{-0.1}\right)$, we estimate the free-free contribution of 4.7 and $8.4 \mathrm{Jy}(\sim 9 \%$ and $24 \%$ of the total flux densities at $1.3 \mathrm{~mm}$ ) at $1.3 \mathrm{~mm}$ toward the continuum cores K1-3 and F1-4 in our observations. Our estimates are consistent with the determinations of Lis et al. (1993; 6\% and 33\% of the total flux densities at $1.3 \mathrm{~mm}$ ) and Martin-Pintado et al. (1990; $\sim 9 \%$ and $28 \%$ of the total flux densities at $1.3 \mathrm{~mm}$ ) for K1-3 and F1-4. The continuum flux densities at $1.3 \mathrm{~mm}$ corrected for free-free emission are 47.4 and 27.2 Jy for K1-3 and F1-4, respectively.

From the flux densities at $1.3 \mathrm{~cm}$ (Gaume et al. 1995), the estimated free-free emission contributions at $1.3 \mathrm{~mm}$ are $0.02 \mathrm{Jy}$, $0.04 \mathrm{Jy}$, and $1.26 \mathrm{Jy}$ for K4, Z10.24, and MW, respectively. The corresponding continuum flux densities corrected for free-free emission are $3.5,9.1$, and $11.0 \mathrm{Jy}$, respectively.

The derived $\mathrm{H}_{2}$ masses, column densities, and number densities are summarized in Table 4 . The estimated $\mathrm{H}_{2}$ masses of the Sgr B2(N) core (K1-3) and the Sgr B2(M) core (F1-4) are larger than those given by Lis et al. (1993), while the $\mathrm{H}_{2}$ number densities are less than their results. This result is caused by the relatively larger size and higher flux densities of the continuum in our observations.

The $\mathrm{H}_{2} \mathrm{CO}\left(3_{03}-2_{02}\right)$ spectra show absorption against both Sgr B2(N) and Sgr B2(M) compact cores and multiple absorbing peaks. The absorptions are dominated by redshifted gas, suggesting that the lower transition $\mathrm{H}_{2} \mathrm{CO}\left(3_{03}-2_{02}\right)$ traces the cold gas in front of the continuum cores falling into the two compact cores.

Previous observations showed multiple massive young stars in Sgr B2(N) and Sgr B2(M) (e.g., Gaume et al. 1995; de Pree et al. 1998). There are multiple absorbing peaks with different optical depths from our $\mathrm{H}_{2} \mathrm{CO}$ spectra in the Sgr B2(N) and Sgr B2(M) 
TABLE 3

LVG Model Results

\begin{tabular}{|c|c|c|c|c|c|c|c|}
\hline Source & Absorption or Emission ${ }^{a}$ & $\begin{array}{c}V_{\mathrm{LSR}} \\
\left(\mathrm{km} \mathrm{s}^{-1}\right)\end{array}$ & $I_{\mathrm{p} 1} / I_{\mathrm{p} 2}$ & Model $^{\mathrm{b}}$ & $\begin{array}{r}T_{k} \\
(\mathrm{~K})\end{array}$ & $\begin{array}{c}n_{\mathrm{H}_{2}} \\
\left(10^{5} \mathrm{~cm}^{-3}\right)\end{array}$ & $\begin{array}{c}\log \left(N_{\text {para- } \mathrm{H}_{2} \mathrm{CO}} / \Delta V\right) \\
\left(\mathrm{cm}^{-2} \mathrm{~km}^{-1} \mathrm{~s}\right)\end{array}$ \\
\hline \multirow[t]{15}{*}{ K1-3 „...................... } & \multirow[t]{5}{*}{ (a) } & \multirow[t]{5}{*}{63} & \multirow[t]{5}{*}{$2.2 \pm 0.2$} & A & $61_{-11}^{+12}$ & $12.6_{-3.3}^{+6.1}$ & $14.9-15.2$ \\
\hline & & & & $\mathrm{B}$ & $135_{-5}^{+5}$ & $17.8_{-0.5}^{+10.4}$ & $15.3-15.6$ \\
\hline & & & & $\mathrm{C}$ & $200_{-70}^{+280}$ & $10.0_{-7.5}^{+10.0}$ & $15.3-15.7$ \\
\hline & & & & $\mathrm{D}$ & $300_{-150}^{+150}$ & $0.22_{-0.09}^{+0.03}$ & $16.5-17.6$ \\
\hline & & & & $\mathrm{E}$ & $255_{-95}^{+95}$ & $0.07_{-0.01}^{+0.02}$ & $17.1-17.3$ \\
\hline & \multirow[t]{5}{*}{ (a) } & \multirow[t]{5}{*}{72} & \multirow[t]{5}{*}{$1.4 \pm 0.1$} & $\mathrm{~A}$ & $61_{-3}^{+4}$ & $71_{-26}^{+245}$ & $15.1-15.2$ \\
\hline & & & & $\mathrm{B}$ & $105_{-35}^{+25}$ & $8.3_{-3.3}^{+5.9}$ & $15.8-16.2$ \\
\hline & & & & $\mathrm{C}$ & $356_{-10}^{+12}$ & $35_{-27}^{+124}$ & $16.3-16.8$ \\
\hline & & & & $\mathrm{D}$ & $80_{-30}^{+120}$ & $0.25_{-0.09}^{+0.07}$ & $17.3-17.4$ \\
\hline & & & & $\mathrm{E}$ & $105_{-50}^{+75}$ & $0.09_{-0.03}^{+0.01}$ & $17.3-17.4$ \\
\hline & \multirow[t]{5}{*}{ (a) } & \multirow[t]{5}{*}{84} & \multirow[t]{5}{*}{$5 \pm 0.3$} & A & $25_{-5}^{+4}$ & $79_{-23}^{+36}$ & $13.7-14.1$ \\
\hline & & & & $\mathrm{B}$ & $34_{-7}^{+5}$ & $316_{-158}^{+1268}$ & $13.8-13.9$ \\
\hline & & & & $\mathrm{C}$ & $30_{-14}^{+14}$ & $316_{-158}^{+2195}$ & $13.5-13.6$ \\
\hline & & & & $\mathrm{D}$ & $200_{-40}^{+20}$ & $0.025_{-0.008}^{+0.007}$ & $17.2-17.3$ \\
\hline & & & & $\mathrm{E}$ & $200_{-100}^{+40}$ & $0.03_{-0.00}^{+0.01}$ & $17.0-17.11$ \\
\hline \multirow[t]{3}{*}{ NE } & \multirow[t]{3}{*}{ (a) } & \multirow[t]{3}{*}{72} & \multirow[t]{3}{*}{$2.4 \pm 0.5$} & $\mathrm{C}$ & $25_{-1}^{+1}$ & $25.0_{-11}^{+476}$ & $14.6-14.8$ \\
\hline & & & & $\mathrm{D}$ & $55_{-10}^{+1}$ & $6.3_{-6}^{+93.7}$ & $14.8-15.8$ \\
\hline & & & & $\mathrm{E}$ & $55_{-10}^{+2}$ & $5.0_{-2.5}^{+26.6}$ & $14.9-15.5$ \\
\hline \multirow[t]{6}{*}{ Z10.24 ................... } & \multirow[t]{3}{*}{ (a) } & \multirow[t]{3}{*}{70} & $3.3 \pm 1.1$ & $\mathrm{C}$ & $26_{-1}^{+1}$ & $10_{-4}^{+30}$ & $14.6-14.8$ \\
\hline & & & & $\mathrm{D}$ & $50_{-20}^{+8}$ & $2.0_{-1.9}^{+23.1}$ & $14.8-15.6$ \\
\hline & & & & $\mathrm{E}$ & $56_{-17}^{+3}$ & $2.5_{-2.3}^{+97.5}$ & $14.7-15.1$ \\
\hline & (e) & 90 & $4.0 \pm 1.3$ & $\mathrm{C}$ & $50_{-11}^{+47}$ & $1.45_{-0.60}^{+0.54}$ & $13.3-14.1$ \\
\hline & & & & $\mathrm{D}$ & $\ldots$ & $\ldots$ & $\ldots$ \\
\hline & & & & E & $60_{-15}^{+120}$ & $0.79_{-0.69}^{+1.72}$ & $13.0-13.9$ \\
\hline 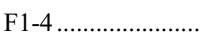 & (a) & 58 & $1.6 \pm 0.2$ & A & $77_{-3}^{+3}$ & $17.8_{-6.9}^{+23.99}$ & $15.4-15.7$ \\
\hline & & & & $\mathrm{B}$ & $132_{-3}^{+2}$ & $10_{-6}^{+22}$ & $15.7-16.0$ \\
\hline & & & & $\mathrm{C}$ & $288_{-8}^{+5}$ & $8.91_{-5.76}^{+41.1}$ & $16.3-17.1$ \\
\hline & & & & $\mathrm{D}$ & $575_{-15}^{+10}$ & $7.08_{-3.92}^{+8.72}$ & $17.1-17.4$ \\
\hline & & & & $\mathrm{E}$ & $260_{-20}^{+10}$ & $0.05_{-0.01}^{+0.01}$ & $17.05-17.15$ \\
\hline & (a) & 62 & $3.4 \pm 0.6$ & A & $40_{-26}^{+26}$ & $7.94_{-4.78}^{+31.8}$ & $14.6-14.9$ \\
\hline & & & & B & $40_{-20}^{+20}$ & $12.6_{-8.6}^{+87.4}$ & $14.4-14.7$ \\
\hline & & & & $\mathrm{C}$ & $180_{-60}^{+30}$ & $0.063_{-0.043}^{+0.078}$ & $16.88-17.04$ \\
\hline & & & & $\mathrm{D}$ & $425_{-25}^{+125}$ & $0.28_{-0.12}^{+0.22}$ & $17.0-17.08$ \\
\hline & & & & $\mathrm{E}$ & $280_{-120}^{+60}$ & $0.04_{-0.01}^{+0.01}$ & $16.86-17.02$ \\
\hline & (a) & 68 & $3.1 \pm 0.5$ & A & $30_{-10}^{+9}$ & $17.8_{-7.8}^{+39.7}$ & $14.60-14.9$ \\
\hline & & & & $\mathrm{B}$ & $41_{-11}^{+10}$ & $31.6_{-19}^{+168}$ & $14.5-14.7$ \\
\hline & & & & $\mathrm{C}$ & $110_{-25}^{+60}$ & $0.10_{-0.08}^{+0.06}$ & $17.1-17.2$ \\
\hline & & & & $\mathrm{D}$ & $465_{-195}^{+35}$ & $0.14_{-0.07}^{+0.48}$ & $17.0-17.18$ \\
\hline & & & & $\mathrm{E}$ & $240_{-100}^{+80}$ & $0.06_{-0.01}^{+0.02}$ & $17.0-17.2$ \\
\hline & (a) & 76 & $5.0 \pm 1.0$ & A & $26_{-10}^{+100}$ & $36.3_{-16.3}^{+122}$ & $14.1-14.2$ \\
\hline & & & & $\mathrm{B}$ & $25_{-12}^{+21}$ & $63.1_{-23.3}^{+137}$ & $13.8-13.92$ \\
\hline & & & & $\mathrm{C}$ & $24_{-1}^{+1}$ & $150_{-79}^{+166}$ & $13.25-13.76$ \\
\hline & & & & $\mathrm{D}$ & $500_{-50}^{+30}$ & $0.018_{-0005}^{+0.032}$ & $16.8-17.1$ \\
\hline & & & & $\mathrm{E}$ & $150_{-70}^{+30}$ & $0.02_{-0.005}^{+0.005}$ & $16.8-16.89$ \\
\hline MW …...................... & (e) & 53 & $1.6 \pm 0.1$ & $\mathrm{C}$ & $34_{-1}^{+1}$ & $4.50_{-1.42}^{+1.81}$ & $15.0-15.16$ \\
\hline & & & & $\mathrm{D}$ & $\ldots$ & $\ldots$ & $\ldots$ \\
\hline & & & & $\mathrm{E}$ & $16_{-1}^{+1}$ & $7.94_{-7.38}^{+7.86}$ & $15.4-15.8$ \\
\hline & (a) & 70 & $3.5 \pm 1.7$ & $\mathrm{C}$ & $28_{-4}^{+1}$ & $3.20_{-1.90}^{+12.6}$ & $14.83-15.77$ \\
\hline & & & & $\mathrm{D}$ & $52_{-22}^{+8}$ & $2.0_{-1.6}^{+77.4}$ & $14.4-14.9$ \\
\hline & & & & $\mathrm{E}$ & $53_{-31}^{+6}$ & $1.68_{-1.52}^{+48.4}$ & $14.9-15.0$ \\
\hline M1 …..................... & (e) & 52 & $1.7 \pm 0.1$ & $\mathrm{C}$ & $35_{-4}^{+5}$ & $3.47_{-1.07}^{+1.54}$ & $15.00-15.16$ \\
\hline & & & & $\mathrm{D}$ & $\ldots$ & $\ldots$ & $\ldots$ \\
\hline & & & & $\mathrm{E}$ & $15_{-1}^{+1}$ & $10.0_{-9.44}^{+15.1}$ & $15.3-16.8$ \\
\hline & (a) & 64 & $1.6 \pm 0.2$ & $\mathrm{C}$ & $25_{-1}^{+1}$ & $3.47_{-1.07}^{+1.54}$ & $15.05-15.1$ \\
\hline & & & & $\mathrm{D}$ & $55_{-4}^{+1}$ & $20_{-9}^{+20}$ & $15.2-16.1$ \\
\hline & & & & $\mathrm{E}$ & $90_{-1}^{+1}$ & $12.6_{-11.3}^{+37.5}$ & $15.2-16.0$ \\
\hline
\end{tabular}


TABLE 3-Continued

\begin{tabular}{|c|c|c|c|c|c|c|c|}
\hline Source & Absorption or Emission ${ }^{a}$ & $\begin{array}{c}V_{\mathrm{LSR}} \\
\left(\mathrm{km} \mathrm{s}^{-1}\right)\end{array}$ & $I_{\mathrm{p} 1} / I_{\mathrm{p} 2}$ & Model $^{b}$ & $\begin{array}{r}T_{k} \\
(\mathrm{~K})\end{array}$ & $\begin{array}{c}n_{\mathrm{H}_{2}} \\
\left(10^{5} \mathrm{~cm}^{-3}\right)\end{array}$ & $\begin{array}{c}\log \left(N_{\text {para- }} \mathrm{H}_{2} \mathrm{CO} / \Delta V\right) \\
\quad\left(\mathrm{cm}^{-2} \mathrm{~km}^{-1} \mathrm{~s}\right)\end{array}$ \\
\hline \multirow[t]{2}{*}{$\mathrm{M} 2 \ldots \ldots \ldots \ldots \ldots \ldots \ldots \ldots$} & (e) & 55 & $5.2 \pm 1.0$ & $\begin{array}{l}\mathrm{C} \\
\mathrm{D}\end{array}$ & $40_{-7}^{+10}$ & $10.0_{-2.4}^{+5.8}$ & $\begin{array}{c}13.45-13.8 \\
\ldots\end{array}$ \\
\hline & & & & $\mathrm{E}$ & $45_{-15}^{+35}$ & $2.82_{-2.19}^{+7.18}$ & $13.00-13.85$ \\
\hline \multirow[t]{2}{*}{$\mathrm{M} 3 \ldots \ldots \ldots \ldots \ldots \ldots \ldots$} & (e) & 53 & $2.4 \pm 0.3$ & $\begin{array}{l}\mathrm{C} \\
\mathrm{D}\end{array}$ & $150_{-50}^{+249}$ & $1.86_{-0.66}^{+0.77}$ & $13.62-13.8$ \\
\hline & & & & $\mathrm{E}$ & $150_{-50}^{+250}$ & $0.45_{-0.31}^{+0.81}$ & $13.30-13.85$ \\
\hline
\end{tabular}

a The (a) and (e) indicate absorption and emission, respectively.

b The filling factors are $f_{C}=0.3, f_{L}=0.4, f_{L, V}=0.25$ for model A, $f_{C}=0.2, f_{L}=0.4, f_{L, V}=0.25$ for model B, $f_{C}=0.1, f_{L}=0.4, f_{L, V}=0.25$ for model C, $f_{C}=0.05, f_{L}=0.4, f_{L, V}=0.25$ for model D, and $f_{C}=0.05, f_{L}=1, f_{L, V}=1$ for model E. For the absorption components, we used the following formula in the radiative transfer calculation:

$$
\Delta T_{L}^{\mathrm{obs}}=\left(f_{L} T_{\mathrm{ex}}-\frac{f_{L}}{f_{C}} T_{C}^{\mathrm{obs}}\right)\left(1-e^{-\tau_{L}}\right) .
$$

For the emission components, we assumed $f_{0}=0$ and used the following formula:

$$
\Delta T_{L}^{\mathrm{obs}}=\left(f_{L} T_{\mathrm{ex}}-T_{\mathrm{cmb}}\right)\left(1-e^{-\tau_{L}}\right),
$$

where $T_{\mathrm{cmb}}$ is the temperature of the cosmic microwave background radiation.

cores, which appears to indicate that the gas is falling into the massive stars or massive star-forming cores embedded at different depths in the molecular clouds (Mehringer et al. 1995). However, the angular resolution of our observations is inadequate for us to determine whether there are multiple regions present or whether the overall gravitational potential dominates the infalling gas. If infalling gas is in simple free fall, the infalling velocities can be estimated by

$$
V_{\text {in }}=\sqrt{\frac{2 M G}{r_{\text {in }}}}=0.09 \sqrt{\frac{M / M_{\odot}}{r_{\text {in }} / \mathrm{pc}}} \mathrm{km} \mathrm{s}^{-1},
$$

where $r_{\text {in }}$ is the infall radius, $M$ is the sum of the gas and star masses included in the $r_{\text {in }}$, and $G$ is the gravitational constant.

The $\mathrm{H}_{2}$ masses derived from the continuum are $1.4 \times 10^{4}$ and $7.9 \times 10^{3} M_{\odot}$ for the cores of Sgr B2(N) and Sgr B2(M), respectively. The Very Large Array observations of radio continuum at $1.3 \mathrm{~cm}$ (Gaume et al. 1995) showed that there are three UCH II regions $(\mathrm{K} 1, \mathrm{~K} 2$, and $\mathrm{K} 3)$ in the core of Sgr B2(N) and four $\mathrm{UCH}$ II regions (F1, F2, F3, and F4) in the core of Sgr B2(M). By use of the relationships between stellar spectral type and stellar mass (Vacca et al. 1996), a total stellar mass of $68 M_{\odot}$ was inferred for the massive stars in the core of $\operatorname{Sgr} B 2(\mathrm{~N})$. The higher resolution observations $\left(0.05^{\prime \prime}\right)$ at $7 \mathrm{~mm}$ (de Pree et al. 1998) resolved

\begin{tabular}{|c|c|c|c|}
\hline Source & $\begin{array}{c}M_{\mathrm{H}_{2}} \\
\left(10^{3} M_{\odot}\right)\end{array}$ & $\begin{array}{c}N_{\mathrm{H}_{2}} \\
\left(10^{24} \mathrm{~cm}^{-2}\right)\end{array}$ & $\begin{array}{c}n_{\mathrm{H}_{2}} \\
\left(10^{6} \mathrm{~cm}^{-3}\right)\end{array}$ \\
\hline$K 1-3^{a} \ldots \ldots$ & $13.7 \pm 1$ & $21.2 \pm 2$ & $18.7 \pm 2$ \\
\hline $\mathrm{K} 4 \ldots \ldots \ldots \ldots \ldots \ldots \ldots \ldots \ldots \ldots \ldots$ & $1.0 \pm 0.1$ & $1.5 \pm 0.1$ & $1.3 \pm 0.1$ \\
\hline NE & $0.73 \pm 0.06$ & $3.2 \pm 0.3$ & $4.8 \pm 0.4$ \\
\hline 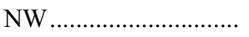 & $0.66 \pm 0.06$ & $1.2 \pm 0.1$ & $1.1 \pm 0.1$ \\
\hline 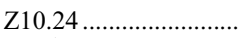 & $2.6 \pm 0.2$ & $1.7 \pm 0.1$ & $0.9 \pm 0.1$ \\
\hline F1-4 & $7.8 \pm 0.8$ & $9.0 \pm 1.3$ & $6.6 \pm 0.7$ \\
\hline 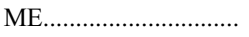 & $0.28 \pm 0.03$ & $1.0 \pm 0.1$ & $2.4 \pm 0.3$ \\
\hline 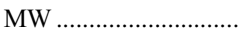 & $3.2 \pm 0.4$ & $1.1 \pm 0.1$ & $0.46 \pm 0.06$ \\
\hline
\end{tabular}

TABLE 4

The Properties of Sgr B2 Derived from Dust Emission

a The core of Sgr B2(N).

b The core of Sgr B2(M). out F1, F2, F3, and F4 into $21 \mathrm{UCH}$ II regions, and a stellar mass of $443 M_{\odot}$ was inferred corresponding to the massive stars in the core of Sgr B2(M). The mass of the massive stars in the Sgr $\mathrm{B} 2(\mathrm{M})$ core is 6 times larger than that in the Sgr B2(N) core. Taking the major axis sizes of 0.24 and $0.29 \mathrm{pc}$ as the infall radii of the two cores (K1-3 and F1-4), we inferred the infall velocities of 21 and $15 \mathrm{~km} \mathrm{~s}^{-1}$ for Sgr B2(N) and Sgr B2(M), respectively. Hence, based on our SMA observations, we have shown that highdensity molecular gas is continuously feeding onto the active star formation cores in both Sgr B2(M) and Sgr B2(N).

\section{SUMMARY}

The continuum emission and $\mathrm{H}_{2} \mathrm{CO}$ spectral lines at $1.3 \mathrm{~mm}$ were observed with the SMA. We detected the continuum emission from the compact cores of Sgr B2(N) and Sgr B2(M). Outside of the two compact cores, a few clumps, K4, NE, NW, ME, $\mathrm{MW}$, and the filament of Z10.24 were also observed. Except for the three newly observed objects, NE, NW, and ME, all of the other compact cores, clumps, and the filament have radio counterparts at centimeter wavelengths.

The spectra in the two $\mathrm{H}_{2} \mathrm{CO}$ lines toward the continuum and outside of the continuum showed the gas components in either emission or absorption. From the integrated line intensity and intensity-weighted velocity maps in line emission, we identified possible outflow motion in the Sgr B2(N) and Sgr B2(M) cores. The infalling gas was detected by the redshifted absorbing gas against the continuum in the Sgr B2(N) and Sgr B2(M) compact cores and the nearby dust clumps and filament Z10.24. The absorbing gas in the Sgr B2(N) and Sgr B2(M) cores is redshifted with respect to the systematic velocities, suggesting that the gas in front of the continuum cores is flowing onto the continuum cores.

In comparison to $\mathrm{Sgr}$ B2(N), the kinematics in Sgr B2(M) are relatively simple. We interpreted the observed outflow and infall in Sgr B2(M) by a model incorporating a spherically symmetric inflow with a decelerating outflow. A decelerating outflow from Sgr B2(M) was evident in our observations. We showed that both the redshifted and blueshifted outflow components share a common terminal velocity. From the outflow terminal velocity, we determined the systematic velocity of $58 \pm 2 \mathrm{~km} \mathrm{~s}^{-1}$ for Sgr B2(M). With the systematic velocity well determined in a manner independent of chemistry in the molecular cloud, we are confident 
that the majority of the absorbing gas at least in Sgr B2(M) is redshifted and flowing toward the active star-forming core.

Using the observed two $\mathrm{H}_{2} \mathrm{CO}$ lines, we have derived the excitation conditions of $\mathrm{Sgr} \mathrm{B} 2$ region in $\mathrm{LVG}$ approximation. For the absorbing components in the Sgr B2(N) and Sgr B2(M) cores, the kinetic temperature, the $\mathrm{H}_{2}$ density, and column density are in the range of several tens to hundreds of $\mathrm{K}, 10^{5-}-10^{7} \mathrm{~cm}^{-3}$, and $10^{14}-10^{17} \mathrm{~cm}^{-2} \mathrm{~km}^{-1} \mathrm{~s}$, respectively. The components outside of the cores are relatively cooler, and the $\mathrm{H}_{2}$ density and column density are 1 order of magnitude less than those in the Sgr B2(N) and Sgr B2(M) cores. In the Sgr B2(M) outflow region, the inten- sity of the $\mathrm{H}_{2} \mathrm{CO}\left(3_{21}-2_{20}\right)$ is larger than that of the $\mathrm{H}_{2} \mathrm{CO}\left(3_{03}-2_{02}\right)$, suggesting the inversion of the distribution of the particle number between the two ground levels of $K_{-1}=0$ and 2. The $\mathrm{H}_{2} \mathrm{CO}\left(3_{21}-2_{20}\right)$ might be excited by $\mathrm{C}$ shocks in the outflow. The radiative excitation by the strong FIR radiation field in Sgr B2(M) may play an important role in the population inversion.

We thank the SMA staff for making the observations possible. J. J. Wang acknowledges support from National Natural Science Foundation of China under grant 10328306.

\section{APPENDIX A}

\section{A MODEL FOR A MOLECULAR CLOUD WITH INFLOW AND DECELERATING OUTFLOW COMPONENTS}

In this appendix we discuss a model incorporating a spherically symmetric inflow (Shu 1977) with a decelerating outflow (Cabrit \& Bertout 1990; Raga et al. 1993). We calculated position-velocity (PV) diagrams based on this infall-outflow model. These PV diagrams can be compared with the observed PV diagrams from Sgr B2(M) to better understand the physical processes in the Sgr B2(M) molecular cloud.

\section{A1. INFALL}

For an ideal isothermal flow, under the assumption of spherically symmetric collapse, the volume density $\rho(r)$ and velocity profiles $V(r)$ as functions of radius $r$ within the collapsing inner envelope can be described by the power laws (Shu 1977),

$$
\rho(r)=\rho_{0}\left(\frac{r}{R}\right)^{-3 / 2}, \quad V(r)=-\left[\frac{2 G M(r)}{r}\right]^{1 / 2},
$$

where $R$ is outer boundary of the collapsing cloud in spherical geometry, $\rho_{0}$ is the density at $R, M(r)$ is the mass of the cloud interior to $r$, and $G$ is the gravitational constant. Because the actual core has a finite size, the infall is terminated at an inner radius of $r_{0}$. The negative sign in the velocity indicates that the direction of the inflow is opposite that of the unit vector of radius in the spherical geometry. The geometry of a cloud with spherical inflow and decelerating outflow is shown in the Cartesian coordinate system $(x, y, z)$ in Figure 9 . The sky plane is the $x-y$ plane.

The column density $(N)$ at a sky position $(x, y)$ can be calculated by integrating the volume density $\rho(r)$. The column density in the sky plane is

$$
N(x, y)=2 \rho_{0} R \int_{0}^{\sqrt{1-a^{2}}}\left(s^{2}+a^{2}\right)^{-3 / 4} d s
$$

where $s=z / R, a=\left[(x / R)^{2}+(y / R)^{2}\right]^{1 / 2}$. In order to get an analytical form for the column density, we express $\left(s^{2}+a^{2}\right)^{-3 / 4}$ in a Taylor expansion. Neglecting the contribution from higher order terms since the interesting region corresponds to $a \ll 1$ [or $\left(x^{2}+y^{2}\right)^{1 / 2} \ll R$ ] in the practical case, the final solution of equation (A2) is

$$
N(x, y) \cong 5 \rho_{0} \frac{R^{3 / 2}}{\left(x^{2}+y^{2}\right)^{1 / 4}}, \quad \sqrt{x^{2}+y^{2}} \neq 0 .
$$

At $(x, y)=(0,0)$, the inflow terminates at the inner boundary $r_{0}$. Assuming the emission from the far-side gas is blocked by the central compact object, the effective column density along the line of sight is

$$
N(0,0)=2 \rho_{0} R\left[\left(\frac{r_{0}}{R}\right)^{-1 / 2}-1\right] .
$$

From equation (A1), excluding the mass of the central compact object, the mass $M(r)$ of the spherically symmetric cloud within $r$ can be derived by

$$
M(r)=\int_{r_{0}}^{r} 4 \pi r^{2} \rho(r) d r=\int_{r_{0}}^{r} 4 \pi r^{2} \rho_{0}\left(\frac{r}{R}\right)^{-3 / 2} d r=\frac{8 \pi \rho_{0} R^{3}}{3}\left[\left(\frac{r}{R}\right)^{3 / 2}-\left(\frac{r_{0}}{R}\right)^{3 / 2}\right]
$$

The mass interior to $R$ is $M_{R} \approx\left(8 \pi \rho_{0} / 3\right) R^{3}$ if $R \gg r_{0}$. Therefore the equation (A5) can be rewritten as

$$
M(r) \cong M_{R}\left(\frac{r}{R}\right)^{3 / 2}
$$




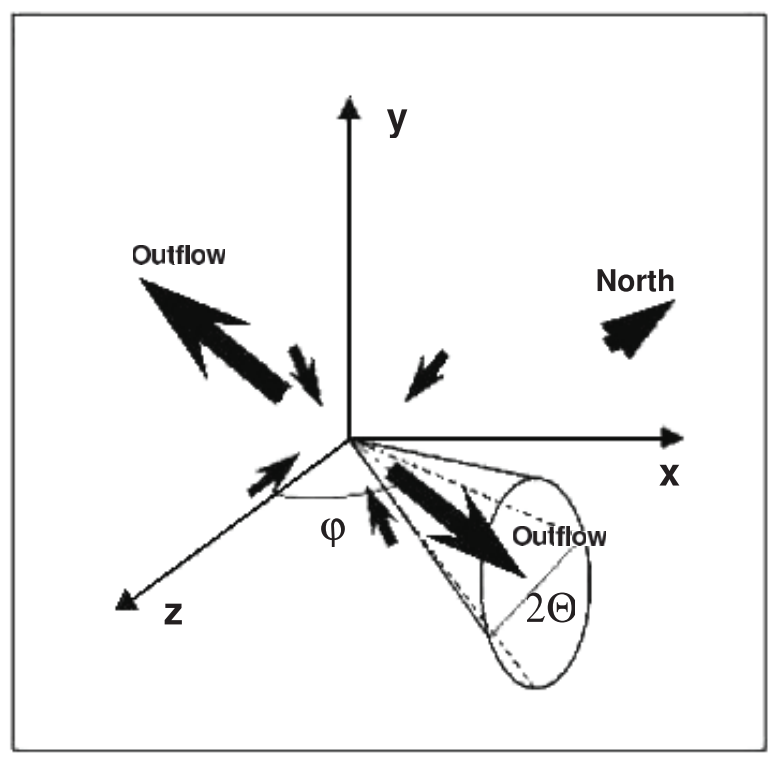

FIG. 9.- Geometry of the layout for a spherical infall (indicated by the small arrows toward the origin of the coordinates) and a decelerating outflow. Here the sky plane is the $x-y$ plane. The outflow lies in the $x-z$ plane with an opening angle of $2 \Theta$ and an inclination angle of $\varphi$. The $z$-axis points toward the observer.

The radial velocity of infall gas is the projection of the velocity vector $\boldsymbol{V}$ on the $z$-axis. The radial velocity averaged along the line of sight is zero at a position away from the central position $(x, y)=(0,0)$ due to the cancellation of the velocity in a spherically symmetric inflow. At the position $(x, y)=(0,0)$, ignoring the contribution of the emission from the region behind the central object, the velocity at $(x, y)=(0,0)$ in front of the central object can be expressed by

$$
V_{\text {infall }}=\frac{\int_{r_{0}}^{R} V(r) d r}{\int_{r_{0}}^{R} d r}=-\frac{\int_{r_{0}}^{R}[2 G M(r) / r]^{1 / 2} d r}{\int_{r_{0}}^{R} d r}=-\frac{4 \sqrt{2}}{5} \sqrt{\frac{G M_{R}}{R}} \frac{1-\left(r_{0} / R\right)^{5 / 4}}{1-r_{0} / R} .
$$

We note that the sign convention in the spherically symmetric description of infalling gas in front of the central object is opposite to the convention of the radial velocity with respect to the central object in the local standard rest (LSR) frame. In addition, in the derivation above, the systematic velocity is assumed to be zero.

\section{A2. DECELERATING OUTFLOW}

Molecular outflows are observed in star formation regions. Various models have been also proposed to interpret the observed outflow morphology (e.g., Raga et al. 1993; Cabrit \& Berout 1990). In this appendix, a PV diagram for a decelerating flow is calculated. The outflow velocity field and density are assumed to have power-law distributions along the major axis of the flow (cf. Cabrit \& Bertout 1990)

$$
V(r)=V_{0}\left(\frac{r_{\min }}{r}\right)^{\alpha}, \quad \rho(r)=\rho_{0}\left(\frac{r_{\min }}{r}\right)^{\delta}
$$

where $\rho_{0}$ and $V_{0}$ are the volume density and velocity at the inner radius $r_{\min }$, respectively; $r=\left(x^{2}+z^{2}\right)^{1 / 2}$, the distance from the center, because the flow is in the $x-z$ panel, i.e., $y=0$. The geometry of the blueshifted outflow is illustrated in Figure 9 by the cone in the $x-z$ plane with an opening angle of $2 \Theta$. The inclination angle of $\varphi$ is the angle between the $z$-axis and the axis of the blueshifted outflow. The angle between the north and $x$-axis is the negative position angle of the blueshifted outflow if we take the convention of position angle in AIPS. The radius of an outflow cross section is equal to $r \tan (\Theta)$. Given conservation of mass flux across a cross section and $0^{\circ}<\Theta<90^{\circ}$, the relationship of the power-law indices $\delta$ and $\alpha$ can be derived

$$
\delta=2-\alpha
$$

The size of a cut across the major axis of the outflow along the line of sight ( $z$-axis) can be expressed approximately by

$$
L_{Z} \approx \tan (\Theta) x / \sin ^{2}(\varphi)
$$

if we take $\alpha=1$ and $\delta=1$ (Cabrit \& Bertout 1990), then the column density along the line of sight can be calculated from

$$
N(x)=\int \rho d z=\int_{0}^{L_{Z}} \rho_{0}\left(\frac{r_{\min }}{r}\right) d z
$$


No. 1, 2008

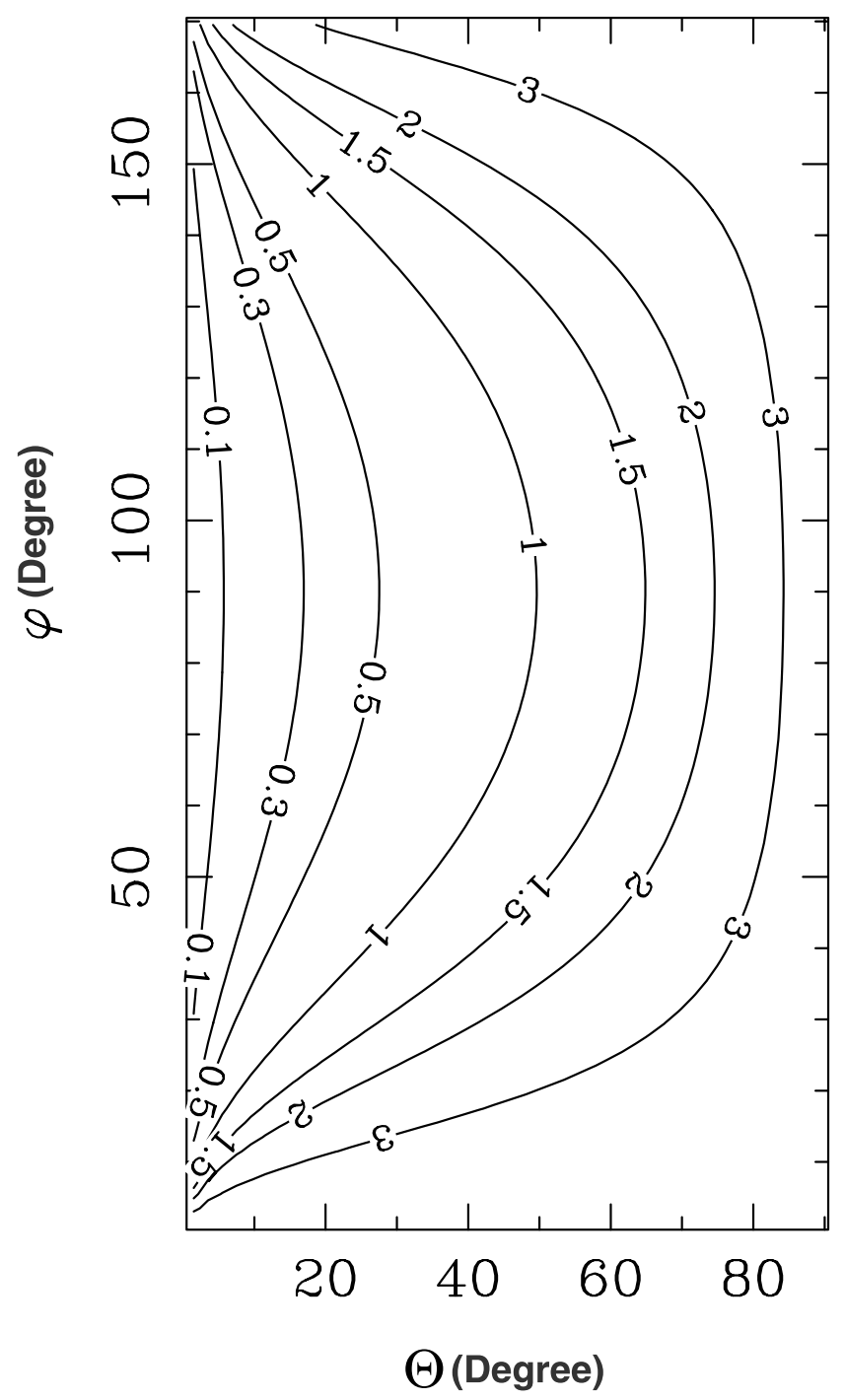

FIG. 10.-Geometric function $g(\Theta, \varphi)$.

the result of the integration is

$$
N(x)=\rho_{0} r_{\min } g(\Theta, \varphi), \quad g(\Theta, \varphi)=\ln \left[\frac{\tan \Theta+\left(\tan ^{2} \Theta+\sin ^{4} \varphi\right)^{1 / 2}}{\sin ^{2} \varphi}\right]
$$

The column density depends on the radius of the inner cross section $\left(r_{\min }\right)$, volume density $\rho_{0}$ at $r_{\min }$, the opening angle $(\Theta)$, and inclination angle $(\varphi)$. In practice, the $\varphi$ and $\Theta$ are in the ranges of $0^{\circ}<\varphi<180^{\circ}$ and $0^{\circ}<\Theta<90^{\circ}$, respectively. A blueshifted outflow corresponds to $0^{\circ}<\varphi<90^{\circ}$ and $90^{\circ}<\varphi<180^{\circ}$ to a redshifted outflow. Figure 10 plots $g(\Theta, \varphi)$ as a function of $\Theta$ and $\varphi$. The value $g(\Theta, \varphi)$ is a weak function of $\Theta$ and $\varphi$. Given $\Theta=30^{\circ}$ and $\varphi=45^{\circ}, g$ is close to unity.

The mean radial velocity of the outflow can be expressed as

$$
V(x)=\frac{\int v(r) \cos \varphi d z}{\int d z}=\frac{V_{0} r_{\min } f(\Theta, \varphi)}{x}, \quad f(\Theta, \varphi)=\frac{g(\Theta, \varphi) \sin ^{2} \varphi \cos \varphi}{\tan \Theta}
$$

The radial velocity of the outflow is inversely proportional to $x$ along the major axis of the outflow and is a weak function $[f(\Theta, \varphi)]$ of $\Theta$ and $\varphi$. As noted in the calculation of the infall velocity, the sign of the velocity used in the equation (A13) is opposite to the convention of the radial velocity with respect to the central source. The velocity of the central source with respect to the LSR, or systematic velocity, is not included here. 


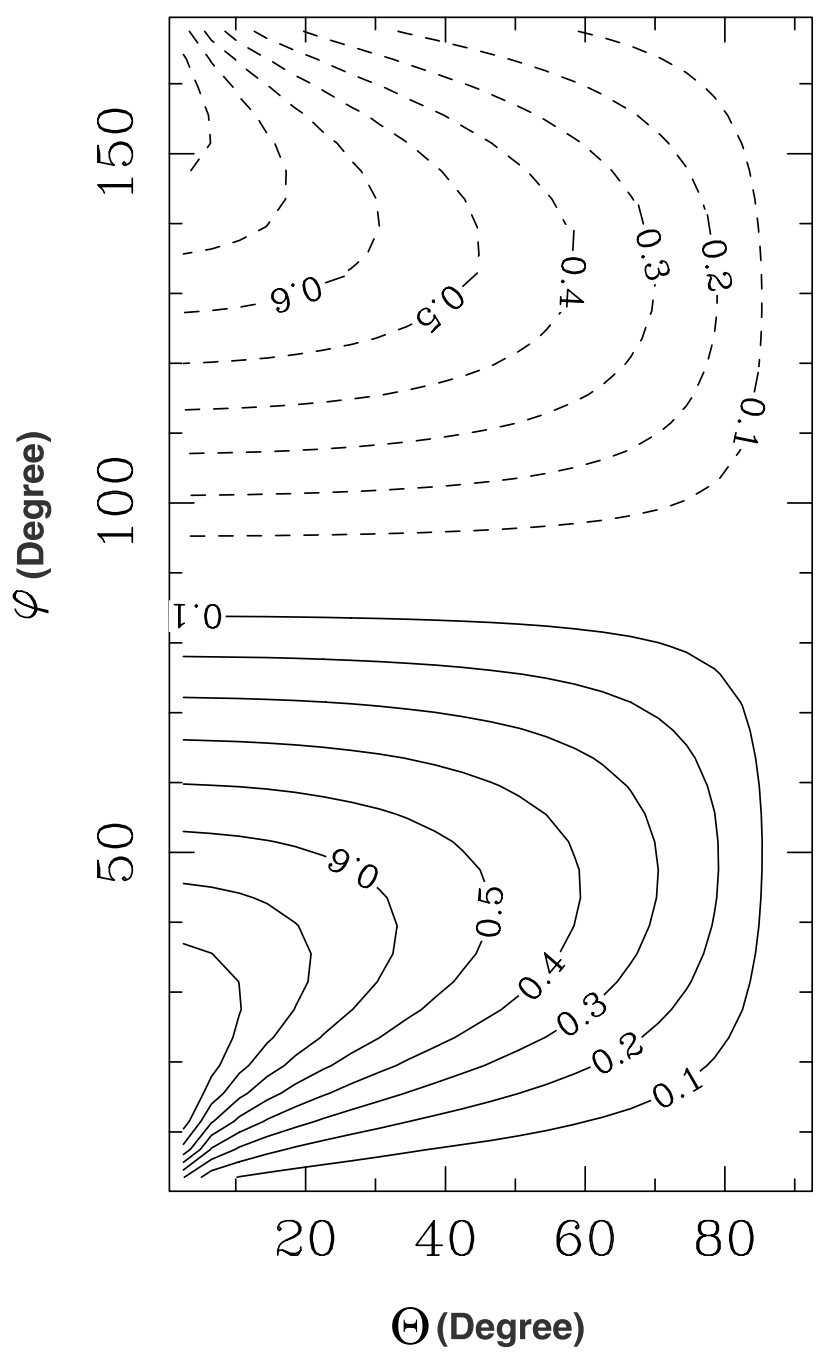

FIG. 11.-Geometric function $f(\Theta, \varphi)$.

Figure 11 shows the plot of $f(\Theta, \varphi)$ as a function of $\Theta$ and $\varphi$. In the range of $90^{\circ}<\varphi<180^{\circ}, f(\Theta, \varphi)$ has a negative value corresponding to a redshifted outflow, while $0^{\circ}<\varphi<90^{\circ}$ is for a blueshifted outflow. Given $\Theta=30^{\circ}$ and $\varphi=45^{\circ}, f$ is about 0.6 . In addition, for given $\varphi, r_{\min }$, and $V_{0}$, the observed radial velocity decreases as $\Theta$ increases.

Assuming $V_{0}=85 \mathrm{~km} \mathrm{~s}^{-1}$ at $r_{0}=1^{\prime \prime}(0.04 \mathrm{pc}), \Theta=30^{\circ}$, and $\varphi=45^{\circ}$, we can calculate a PV diagram of an outflow (the thick solid contour in Fig. 12). Figure 12 shows a PV diagram for the parameter $V_{0} r_{\min } f(\Theta, \varphi)$ in a range between 0.5 and $5.1 \mathrm{~km} \mathrm{~s} \mathrm{~s}^{-1} \mathrm{pc}$, assuming that the blue and redshifted outflows are symmetric around the central source, whose velocity is $58 \mathrm{~km} \mathrm{~s}^{-1}$ with respect to the LSR. In this diagram, we also include an absorption component of a spherical inflow with a mean infall velocity of $8 \mathrm{~km} \mathrm{~s}{ }^{-1}$ with respect to the central source. The PV diagram (Fig. 12) shows the typical configuration of a spherically inflow and decelerating outflows in an active star formation region.

\section{APPENDIX B}

\section{RADIATIVE TRANSFER WITH LVG APPROXIMATION}

In order to model the excitation conditions and the physical properties of the molecular cloud components observed in Sgr B2, we solve for the radiation transfer in a multilevel system with the large velocity gradient (LVG) approximation. In this model, a molecular

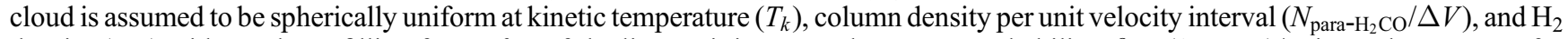
density $\left(n_{\mathrm{H}_{2}}\right)$ with a volume filling factor $f_{L, V}$ of the line-emitting gas. The escape probability, $\beta=\left(1-e^{-\tau}\right) / \tau$, is used to account for photon trapping. The volume filling factor $f_{L, V}$ is incorporated into the opacity calculation via the equation

$$
\tau_{0}=\frac{A_{21}}{8 \pi \nu^{3}} \frac{X_{\mathrm{para}-\mathrm{H}_{2} \mathrm{CO}} n_{\mathrm{H}_{2}} f_{L, V}}{d v / d z}\left(x_{1} \frac{g_{2}}{g_{1}}-x_{2}\right)
$$




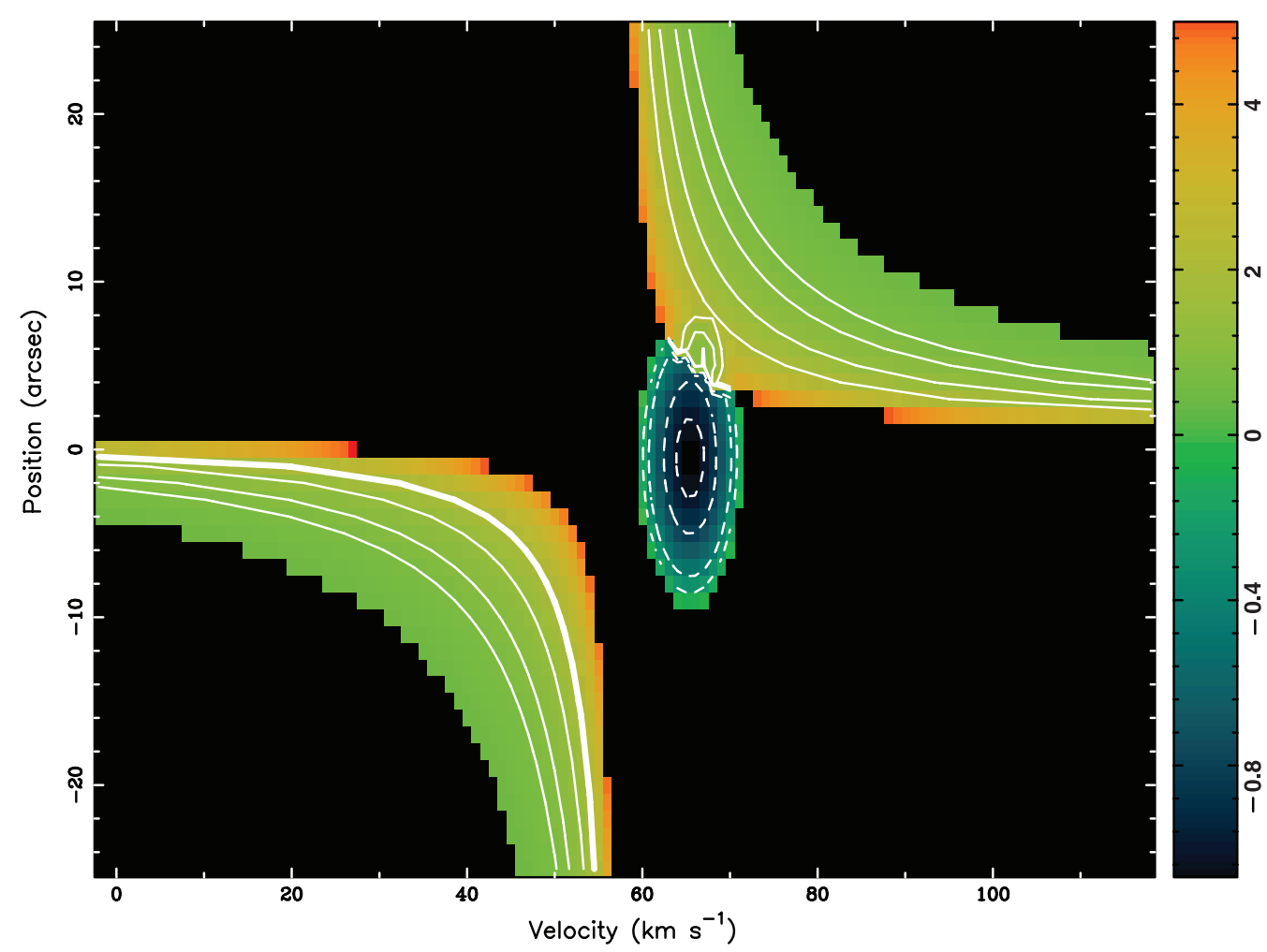

FIG. 12.- The PV diagram calculated from a model incorporating a spherical inflow with decelerating bipolar outflow for the parameter $V_{0} r_{\text {min }} f(\Theta, \varphi)$ in a range between 0.5 and $5.1 \mathrm{~km} \mathrm{~s}^{-1} \mathrm{pc}$ (scaled in color). The position axis (vertical) is a cut along the major axis of the outflow. The velocity axis ( horizontal) is the radial velocity of the outflow with respect to the LSR. The systematic velocity of the central object is assumed to be $58 \mathrm{~km} \mathrm{~s}^{-1}$ and the mean infall velocity of the absorption component is $8 \mathrm{~km} \mathrm{~s}^{-1}$. The contours correspond to $V_{0} r_{\min } f(\Theta, \varphi)=0.8,1,1.4$, and $2 \mathrm{~km} \mathrm{~s}^{-1}$ pc. The thick contour in the blueshifted outflow corresponds to $2 \mathrm{~km} \mathrm{~s} \mathrm{~s}^{-1}$ pc. The absorption (dashed contours) feature at $V=66 \mathrm{~km} \mathrm{~s}^{-1}$ and $X=0^{\prime \prime}$ shows the component of spherical inflow. The normalized absorption intensity is scaled in color.

where $\tau_{0}$ is the optical depth at the line center, $A_{21}$ is the Einstein coefficient for spontaneous emission, $X_{\text {para- }} \mathrm{H}_{2} \mathrm{CO}$ is the abundance of para- $\mathrm{H}_{2} \mathrm{CO}, d v / d z$ is the velocity gradient, and $x_{i}$ and $g_{i}$ are the fractional population and statistical weight of level $i$, respectively. The column density per unit velocity interval at the line center in the LVG model is given by

$$
N_{\text {para- } \mathrm{H}_{2} \mathrm{CO}} / \Delta V=\frac{X_{\text {para- } \mathrm{H}_{2} \mathrm{CO}} n_{\mathrm{H}_{2}} f_{L, V}}{d v / d z}
$$

Given $n_{\mathrm{H}_{2}}, T_{k}$ and $N_{\text {para- } \mathrm{H}_{2} \mathrm{CO}} / \Delta V$, the local LVG model gives the radiative temperature or the intensity of the line requiring as input the collisional excitation rates. We adopted the collision rates calculated by Green (1991). Green used the interaction potential between $\mathrm{H}_{2} \mathrm{CO}$ and $\mathrm{He}$. Since $\mathrm{He}$ has twice the mass of $\mathrm{H}_{2}$, collisional excitation due to $\mathrm{H}_{2}$ should be 2.2 times more effective than collisional excitation by He (Green 1991; Mangum \& Wootten 1993). The collisional excitation rates from Green were multiplied by this factor in our calculations. The accuracy in the Green's calculation of the total collisional excitation rates is $\sim 20 \%$.

Incorporating Green's collisional excitation rates and the volume filling factor discussed above, we used the radiative transfer code in Miriad to solve for the radiative intensities from the para- $\mathrm{H}_{2} \mathrm{CO}$ for the two transitions $3_{03}-2_{02}$ and $3_{21}-2_{20}$. In the line emission case, the line intensity ratio of the two transitions is a good indicator of the kinetic temperature in molecular clouds (e.g., Mangum \& Wootten 1993). This ratio becomes strongly dependent on the volume density $\left(n_{\mathrm{H}_{2}}\right)$ when the kinetic temperature is greater than the upper state energy of the highest excitation transition $\left(E_{u}\right)$. Given a column density $\left(N_{\text {para- } \mathrm{H}_{2} \mathrm{CO}} / \Delta V\right)$, both the intensity of the lower transition $I\left(3_{03}-2_{02}\right)$ and the intensity ratio $\left[I\left(3_{03}-2_{02}\right) / I\left(3_{21}-2_{20}\right)\right]$ as a function of $T_{k}$ and $n_{\mathrm{H}_{2}}$ can be calculated. We calculate five models with different sets of volume filling factor of the para- $\mathrm{H}_{2} \mathrm{CO}$ gas $\left(f_{L, V}\right)$, beam filling factor of the line emission or absorption $\left(f_{L}\right)$, and beam filling factor of the continuum emission $\left(f_{C}\right)$. In models $\mathrm{A}, \mathrm{B}, \mathrm{C}$, and $\mathrm{D}$, the line filling factors $f_{L, V}=0.25$ and $f_{L}=0.4$ are assumed, while $f_{L, V}=1$ and $f_{L}=1$ are assumed in model E. The continuum filling factors of $f_{C}=0.3,0.2,0.1,0.05$, and 0.05 are assumed in models A, B, C, D, and E, respectively. Figure 13 shows the LVG results calculated with model C for the component $\mathrm{K} 1-3$ at $72 \mathrm{~km} \mathrm{~s}{ }^{-1}$. The absorption line intensities of the two transitions $3_{03}-2_{02}$ and $3_{21}-2_{20}$ are plotted as a function of the kinetic temperature and $\mathrm{H}_{2}$ density. The line intensity ratio $I\left(3_{03}-2_{02}\right) / I\left(3_{21}-2_{20}\right)$ is also shown (bottom panel). In the high-density and cold region (see the right bottom corner), both transitions becomes optically thick and the excitation temperature is much smaller than the brightness temperature of the background continuum radiation and the line ratio becomes unity. When the gas becomes hot and less dense (left top corner), the line ratio becomes large. Fitting the observed results of the line intensity and the intensity ratio to the LVG model, we can determine $T_{k}$ and $n_{\mathrm{H}_{2}}$. We find that the absorption case is different from the case of emission, and the absorption line intensity of the lower energy transition, $I\left(3_{03}-2_{02}\right)$, is a good indicator of the kinetic temperature $\left(T_{k}\right)$ of a cloud component while the line intensity ratio $I\left(3_{03}-2_{02}\right) / I\left(3_{21}-2_{20}\right)$ places a strong constraint on the $\mathrm{H}_{2}$ volume density $\left(n_{\mathrm{H}_{2}}\right)$ (see the top panel of Fig. 14). 


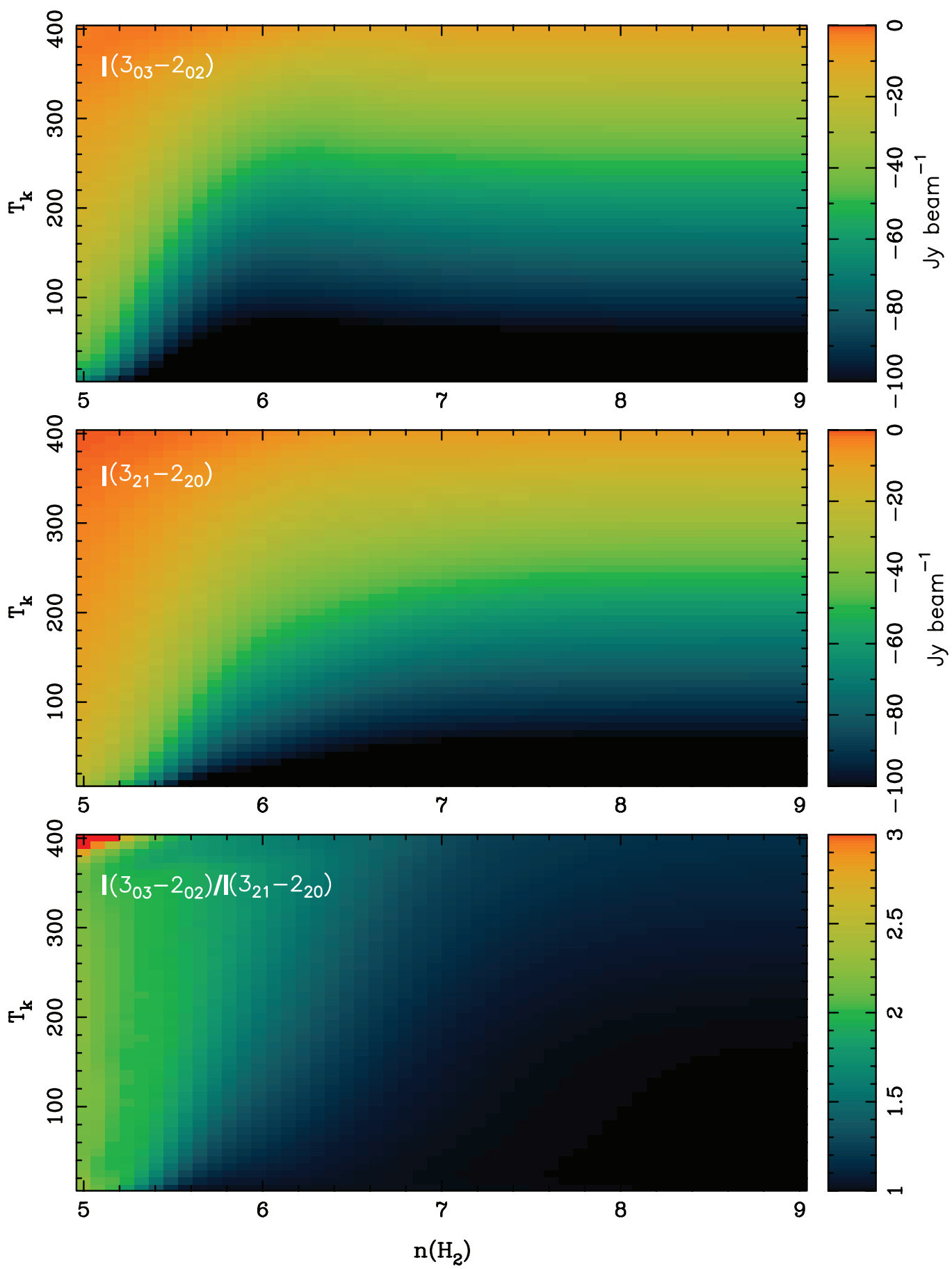

FIG. 13. - Example showing the LVG analysis results derived with model $\mathrm{C}$ for component $\mathrm{K} 1-3$ at $72 \mathrm{~km} \mathrm{~s}^{-1}$. Top: Line intensity of para- $\mathrm{H}_{2} \mathrm{CO}$ transition $I\left(3_{03}-2_{02}\right)$ as a function of $n_{\mathrm{H}_{2}}$ and $T_{k}$. Middle: Line intensity of para- $\mathrm{H}_{2} \mathrm{CO}$ transition $I\left(3_{21}-2_{20}\right)$ as a function of $n_{\mathrm{H}_{2}}$ and $T_{k}$. Bottom: Line ratio of $I\left(3_{03}-2_{02}\right) / I\left(3_{21}-2_{20}\right)$ as a function of $n_{\mathrm{H}_{2}}$ and $T_{k}$.

Figure 14 shows examples of the LVG fitting with model $\mathrm{C}\left(f_{L, V}=0.25, f_{L}=0.4\right.$, and $\left.f_{L, V}=0.1\right)$ to the observed results from three typical regions. The top panel in Figure 14 is the result showing the LVG model curves fitting to the observed $I\left(3_{03}-2_{02}\right)$ and $I\left(3_{03}-2_{02}\right) / I\left(3_{21}-2_{20}\right)$ for the velocity component at $72 \mathrm{~km} \mathrm{~s}^{-1}$ in the region $\mathrm{K} 1-3$ of Sgr B2(N). The dashed lines are absorption in the units of Jy beam ${ }^{-1}$. The thick dashed line and two thin dashed lines in light blue are the observed values of $I\left(3_{03}-2_{02}\right)$ and $1 \sigma$ uncertainty. The solid lines are the intensity ratio $I\left(3_{03}-2_{02}\right) / I\left(3_{21}-2_{20}\right)$. The thick line and two thin lines in red correspond to the observed ratio and $1 \sigma$ uncertainty. The hatched region marks the solution ranges of $2.9 \times 10^{6}-7.9 \times 10^{6} \mathrm{~cm}^{-3}$ in $n_{\mathrm{H}_{2}}$ and $T_{k}=350-$ $360 \mathrm{~K}$ for given $\log \left(N_{\text {para- }} \mathrm{CO} / \Delta V\right)=16.4 \mathrm{~cm}^{-2} \mathrm{~km}^{-1} \mathrm{~s}$. We searched for LVG solutions in a wide range of $11.0-19.0 \mathrm{~cm}^{-2} \mathrm{~km}^{-1} \mathrm{~s}$ in $\log \left(N_{\text {para- }} \mathrm{H}_{2} \mathrm{CO} / \Delta V\right)$. 

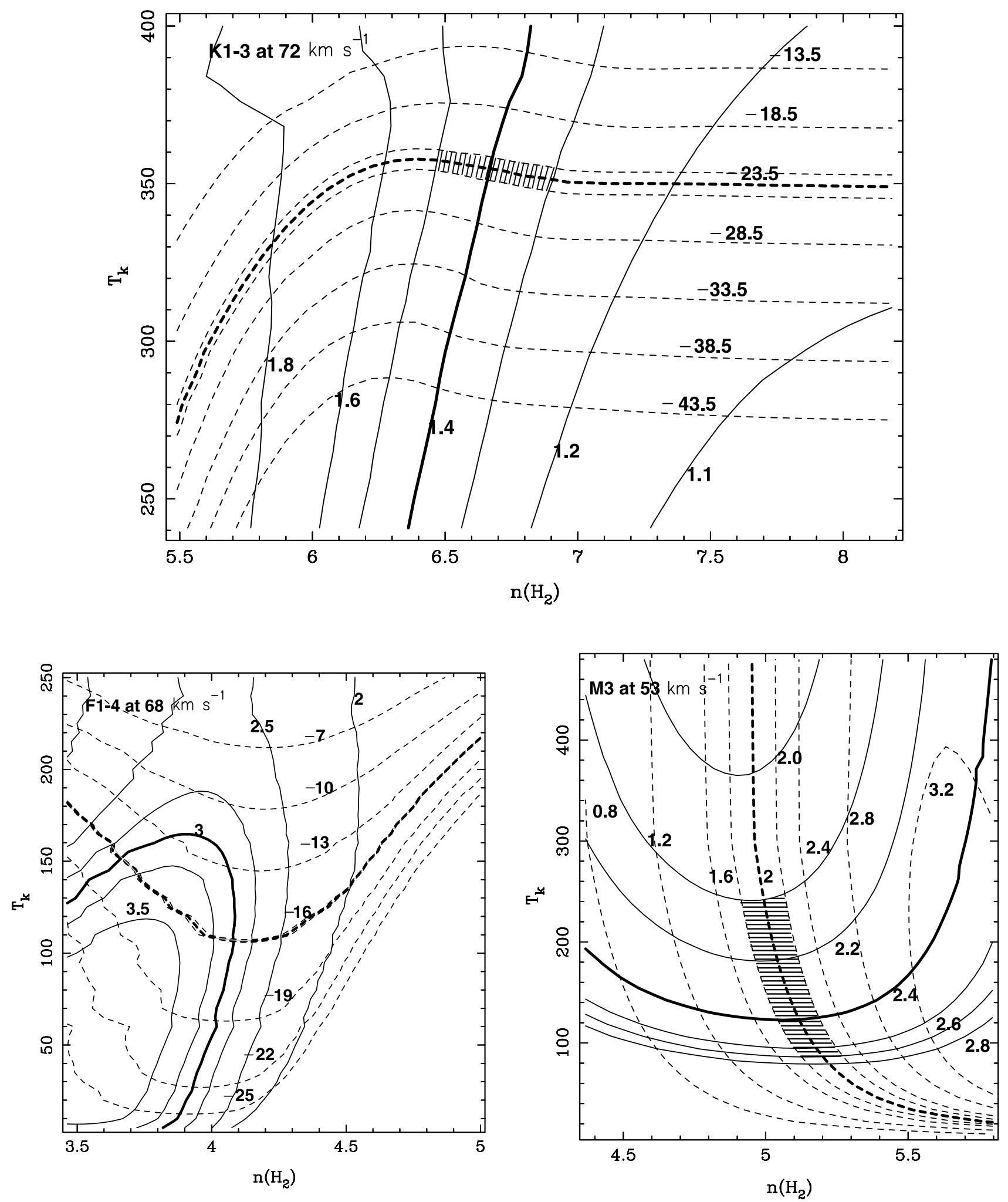

FIG. 14. - Examples of fitting the observed line intensity and intensity ratio to model $\mathrm{C}$ with the LVG approximation. Dashed lines are the line intensity of the $3_{03}-2_{02}$ transition. The solid lines are the line ratio $I\left(3_{03}-2_{02}\right) / I\left(3_{21}-2_{20}\right)$. The thick solid and dashed lines correspond to the observed values of $I\left(3_{03}-2_{02}\right)$ and $I\left(3_{03}-2_{02}\right) / I\left(3_{21}-2_{20}\right)$. The hashed gray zones mark the solution regions in the $n_{\mathrm{H}_{2}}-T_{k}$ domain. Top: Absorption component K1-3 at $72 \mathrm{~km} \mathrm{~s}^{-1}$. Bottom left: Absorption component F1-4 at $68 \mathrm{~km} \mathrm{~s}^{-1}$. Bottom right: Emission component M3 at $53 \mathrm{~km} \mathrm{~s}^{-1}$. [See the electronic edition of the Journal for a color version of this figure.] 
The bottom-left panel in Figure 14 shows the velocity component at $68 \mathrm{~km} \mathrm{~s}^{-1}$ in the F1-4 region of Sgr B2(M). Given $\log \left(N_{\text {para- } \mathrm{H}_{2} \mathrm{CO}} / \Delta V\right)=17.0 \mathrm{~cm}^{-2} \mathrm{~km}^{-1} \mathrm{~s}$, two possible solutions were found for this region, one with higher temperature $\left(T_{k}=150 \mathrm{~K}\right)$ and lower density $n_{\mathrm{H}_{2}}=4.6 \times 10^{3} \mathrm{~cm}^{-3}$ and other with lower temperature $\left(T_{k}=110 \mathrm{~K}\right)$ and higher density $n_{\mathrm{H}_{2}}=1.2 \times 10^{4} \mathrm{~cm}^{-3}$.

The bottom-right panel in Figure 14 shows a LVG model to the observed results from the isolated emission region (M3) at $53 \mathrm{~km} \mathrm{~s}{ }^{-1}$. Given $\log \left(N_{\text {para- }} \mathrm{H}_{2} \mathrm{CO} / \Delta V\right)=13.7 \mathrm{~cm}^{-2} \mathrm{~km}^{-1} \mathrm{~s}, T_{k}=90-240 \mathrm{~K}$ and $n_{\mathrm{H}_{2}}=(0.79-2.3) \times 10^{5} \mathrm{~cm}^{-3}$ were found. The large uncertainties in $T_{k}$ and $n_{\mathrm{H}_{2}}$ are due to relatively weaker line emission.

Cabrit, S., \& Bertout, C. 1990, ApJ, 348, 530

Carlstrom, J. E., \& Vogel, S. N. 1989, ApJ, 337, 408

de Pree, C. G., Gaume, R. A., Goss, W. M., \& Claussen, M. J. 1995, ApJ, 451, 284

1996, ApJ, 464, 788

de Pree, C. G., Goss, W. M., \& Gaume, R. A. 1998, ApJ, 500, 847

de Vicente, P., Martin-Pintado, J., Neri, R., \& Colom, P. 2000, A\&A, 361, 1058

Evans, N. J., II, Davis, J. H., \& Plambeck, R. L. 1979, ApJ, 227, L25

Gaume, R. A., \& Claussen, M. J. 1990, ApJ, 351, 538

Gaume, R. A., Claussen, M. J., de Pree, C. G., Goss, W. M., \& Mehringer, D. M. 1995, ApJ, 449, 663

Goicoechea, J. R., Rodriguze-Fernandez, N. J., \& Cernicharo, J. 2004, ApJ, 600,214

Gordon, M. A. 1995, A\&A, 301, 853

Green, S. 1991, ApJS, 76, 979

Hildebrand, R. H. 1983, QJRAS, 24, 267

Kahane, C., Lucas, R., Frerking, M. A., Langer, W. D., \& Encrenaz, P. 1984, A\&A, 137, 211

Kuan Y.-J., Mehringer, D. M., \& Snyder, L. E. 1996, ApJ, 459, 619

Kuan Y.-J., \& Snyder, L. E. 1994, ApJS, 94, 651 1996, ApJ, 470, 981

Lis, D. C., Carlstrom, J. E., \& Keene. J. 1991, ApJ, 380, 429

Lis, D. C., Goldsmith, P. F., Carlstrom, J. E., \& Scoville, N. Z. 1993, ApJ, 402, 238

Liu, Sheng-Yuan \& Snyder, L. E. 1999, ApJ, 523, 683

Lizano, S., Heiles, C., Rodriguez, L. F., Koo, B.-C., Shu, F. H., Hasegawa, T., Hayashi, S., \& Mirabel, I. F. 1988, ApJ, 328, 763

\section{REFERENCES}

Lucy, L. B. 1971, ApJ, 163, 95

Mangum, J. G., \& Wootten, A. 1993, ApJS, 89, 123

Martin-Pintado, J., de Vicente, Wilson, T. L., \& Johnston, K. J. 1990, A\&A, 236, 193

Mehringer, D. M., Goss, W. M., \& Palmer, P. 1994, ApJ, 434, 237

Mehringer, D. M., Palmer, P., \& Goss, W. M. 1995, ApJS, 97, 497

Mehringer, D. M., Palmer, P., Goss, W. M., \& Yusef-Zadeh, F. 1993, ApJ, 412, 684

Miao, Y. T., Mehringer, D. M., Kuan, Yi-Jheng, \& Snyder, L. E. 1995, ApJ, 445, L59

Nummelin, A., Bergman, P., Hjalmarson, A., Friberg, P., Irvine, W. M., Millar, T. J., Ohishi, M., \& Saito, S. 1998, ApJS, 117, 427

Pierce-Price, D., et al. 2000, ApJ, 545, L121

Raga, A. C., Canto, J., Calvet, N., Rodriguez, L. F., \& Torrelles, J. M. 1993, A\&A, 276, 539

Reid, M. J., Schneps, M. H., Moran, J. M., Gwinn, C. R., Genzel, R., Downes, D., \& Roennaeng, B. 1988, ApJ, 330, 809

Scoville, N. Z., \& Solomon, P. M. 1974, ApJ, 187, L67

Shu, F. 1977, ApJ, 214, 488

Sutton, E. C., Blake, G. A., Masson, C. R., \& Phillips, T. G. 1985, ApJS, 58, 341

Sutton, E. C., Jaminet, P. A., Danchi, W. C., \& Blake, G. A. 1991, ApJS, 77, 255

Vacca, W., Garmany, C., \& Shull, J. 1996, ApJ, 460, 914

Vogel, S. N., Genzel, R., \& Palmer, P. 1987, ApJ, 316, 243 